\title{
Experimental and finite element analyses on stress intensity factors of an elliptical surface crack in a circular shaft under tension and bending
}

\author{
C.S. SHIN* and C.Q. CAI \\ Department of Mechanical Engineering, National Taiwan University. No.1, Sec.4, Roosevelt Road, Taipei, \\ 10617, Taiwan, Republic of China \\ *Author for corresponding (E-mail address: csshin@ntu.edu.tw; Phone +886-2-2362-2160; Fax: \\ $+886-2-2362-2160)$
}

Received 30 September 2003; accepted in revised form 26 April 2004

\begin{abstract}
Experimental backtracking technique and finite element analysis have been employed to evaluate the stress intensities along the front of an elliptical surface crack in a cylindrical rod. The finite element solution covers a wide range of crack shapes loaded under end-free and end-constrained axial tension and pure bending. Convenient closed form stress intensity expressions along the whole crack front for each of the loading cases have been given in terms of the crack aspect ratio, crack depth ratio and place ratio.

The closed form solutions have been compared against a number of representative solutions collected from the literature. It has been found that different finite element results for the interior points are generally in good mutual agreement, while solutions derived from other methods may sometimes indicate different trends. At the surface interception point agreement is less good because of a complication in the interpretation of stress intensity there.

Experimental backtracking results on the end-constrained axial tension case corroborate well with the closed form solution presented. It suggests that the current closed form solution is adequate in describing the stress intensities along the whole crack front of real surface cracks in cylindrical rods.
\end{abstract}

Key words: Closed form stress intensity solution, experiment backtracking technique, stress intensity factor, surface crack.

\section{Introduction}

Cylindrical shaped components such as pins, bolts, reinforcement wires and shafts are commonly used in engineering structures. Under repeated loading, cracks may develop at the surface and grow across the section. Recently, it has been shown that miniature surface cracked rod specimen offer a cost-effective way for evaluating fatigue crack propagation properties (Shin and Chen, 2004). To assess the crack growth behavior and structural integrity involving these cracks, their stress intensity factor solutions must be known. The three-dimensional nature of this kind of cracks results in a stress intensity that is not only varying along the crack front but is also highly sensitive to the crack shape. Numerical techniques or approximate analyses were often employed to estimate the stress intensity for this problem.

Early attempts used a straight edge (Daoud et al., 1978; Bush, 1981; Carpinteri, 1992a) or a circular arc to idealize the crack front (Wilhem et al., 1982; Mackay and Alperin, 1985; Daoud and Cartwright, 1985; Forman and Shivakumar, 1986; Raju and Newman, 1986). In some works, the angle of intersection of the crack front with the rod external surface was taken to be 90o to facilitate crack shape definition (Forman and Shivakumar, 1986; Raju and 
Newman, 1986). These idealizations, though close to, do not as a matter of fact exactly agree with experimental observations. The above discrepancies may have impact on the correctness of the stress intensity solutions. This problem of crack shape description is largely solved by using an elliptical arc to model the crack front (Athanassiadis, 1981; Astiz, 1986; Shiratori et al., 1986; Murakami and Tsuru, 1987; Caspers et al., 1990; Carpinteri, 1992b; Carpinteri and Brighenti, 1996; Couroneau and Royer, 1998; Shih and Chen, 2002). It is well known that the singularity power at the intersection points is no longer $-1 / 2$ and is dependent on the angle of intersection and Poisson ratio (Bazant and Estenssoro, 1979; Hayashi and Abe, 1980; Carpinteri and Brighenti, 1996). The departure from the square root singularity was sometimes pragmatically overcome by discarding the numerical solution at the end point and replaced it with that for a neighboring interior point instead (Carpinteri, 1992b; Carpinteri and Brighenti 1996; Couroneau and Royer, 1998).

Available stress intensity solutions from the literatures for surface cracks in cylindrical rods have some limitations. For example, the reported solutions are often limited to the deepest interior point (Daoud et al., 1978; Daoud and Cartwright, 1985; Bush, 1981; Wilhem et al., 1982; Mackay and Alperin, 1985; Forman and Shivakumar, 1986; Murakami and Tsuru, 1987; Couroneau and Royer, 1998) or the deepest interior and the surface interception points on the crack front (Carpinteri, 1992b; Carpinteri and Brighenti, 1996; Astiz, 1986; Athanassiadis, 1981; Shiratori et al., 1986; Caspers et al., 1990). Sometimes, they are available for a limited number of discrete aspect ratios and crack depth ratios. A closed form solution for the whole crack front covering a wide range of crack geometry is essential for fatigue life evaluation and structural integrity assessment involving surface cracks because practical surface cracks may come in any aspect ratios and crack depths, and such parameters may change as the crack grows along. A closed form solution has recently been published (Shih and Chen, 2002), and covers a wide range of crack aspect ratios and depth ratios, but the present authors think that it is not completely valid (Cai and Shin, 2004a).

In the present work, the stress intensity factors along the crack front are computed for an elliptical surface crack in a cylindrical rod under tension and bending. A wide range of crack aspect ratios that should be able to cover most practical crack shapes is examined. Closed-form equations are given to facilitate use. Moreover, the end condition of practical cylindrical structures under axial tension is often somewhere between unconstrained and fully constrained. These two extreme conditions are dealt with. Finally, experimental verification of the current solution is presented.

\section{Stress intensity factor calculation}

In the present paper, stress intensity factors of the surface cracks in a rod are deduced from finite element stress analysis results via the virtual crack extension method (Parks, 1974; Hellen, 1975). The finite element package ABAQUS has been employed for analysis. Owing to symmetry, only one quarter of the rod has been analyzed. Collapsed quarter point elements (Barsoum, 1976) have been used along the surface crack tip. Figure 1 shows details of the finite element mesh. Axial tensile stress has been applied to the end of the rod. Two kinds of boundary conditions have been considered: an end-constrained case and an end-free case. In the former, the last layer of elements at the end of the rod has been constrained to move in the axial direction. The element dimensions, especially that in the axial direction, have been gradually refined until the solution converged to a consistent value. For the end-constrained 


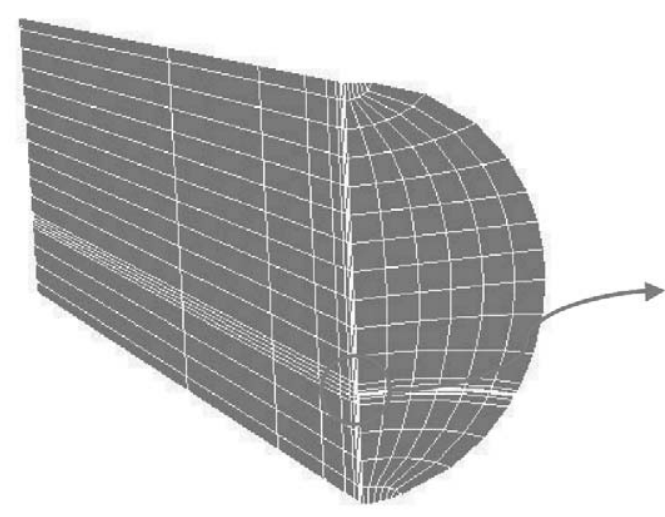

(a) Element mesh

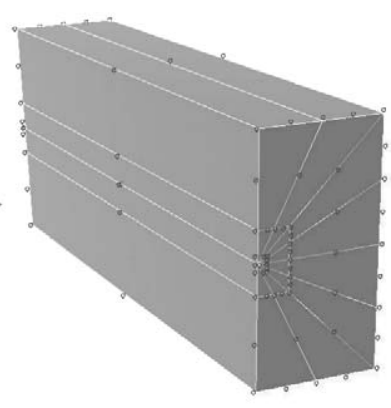

(b) Singularity element

Figure 1. Finite element mesh employed in the current work.

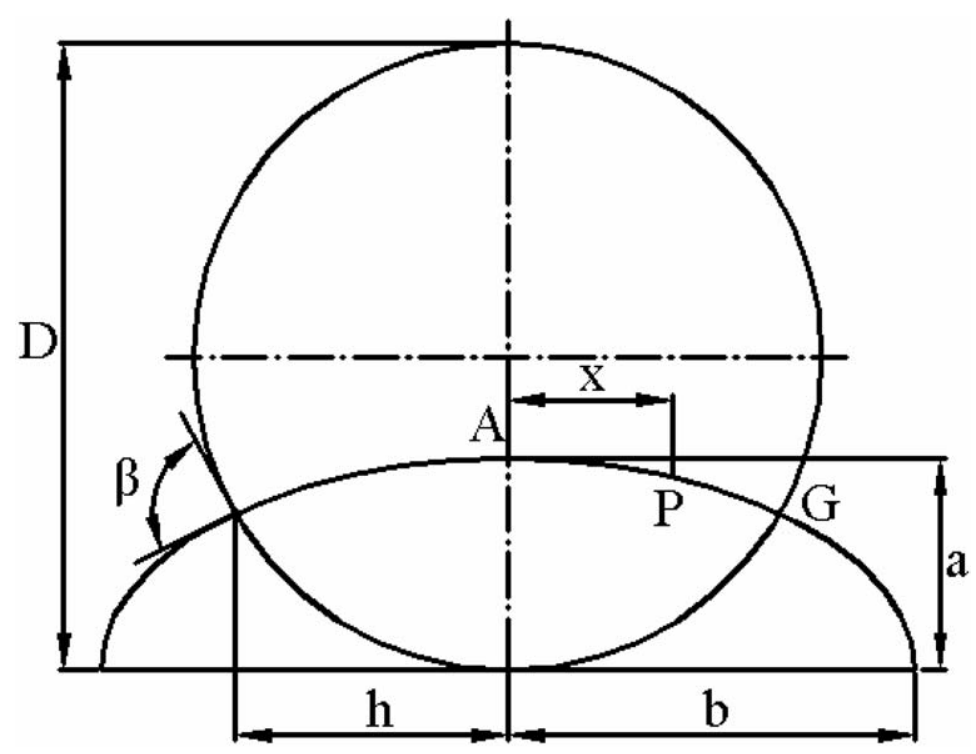

Figure 2. Nomenclature used for an elliptical surface crack.

case, the length of constrained region also affected solution convergence. The constrained end length chosen is $1 \mathrm{~mm}$ for a half-rod length of $90 \mathrm{~mm}$.

\section{Stress intensity solutions}

With the geometrical dimensions defined in Figure 2, the stress intensity $\left(K_{I}\right)$ of the surface crack can be expressed in the following form:

$$
K_{I}=F_{I} \sigma \sqrt{\pi a},
$$

where $\sigma$ is the uniform axial stress or maximum bending stress, $a$ is the crack depth and $F_{I}$ is the geometry correction factor. Since the stress intensity factor of a surface crack is dependent upon the crack shape and varies from point to point on the crack front, $F_{I}$ may be written as: 

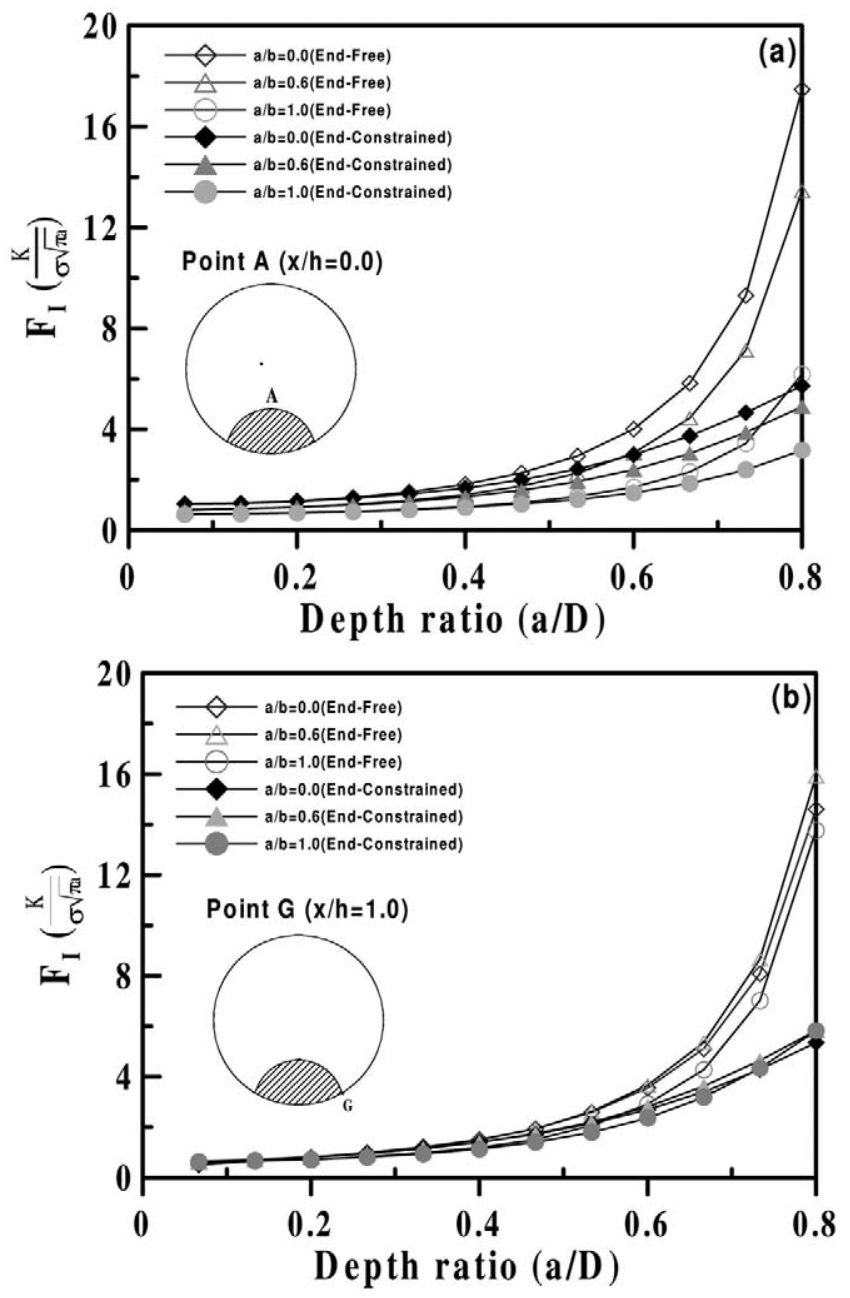

Figure 3. Comparison of some typical results for the end-free and the end-constrained case for selected crack aspect ratios. (a) at the deepest interior point A (b) at the surface point G.

$$
F_{I}=F_{I}\left(\frac{a}{b}, \frac{a}{D}, \frac{x}{h}\right)
$$

where $\frac{a}{b}$ represents the crack aspect ratio, $\frac{a}{D}$ is the crack depth ratio and $\frac{x}{h}$ is the place ratio for identifying different points on the crack front.

Surface cracks with the aspect ratio $\frac{a}{b}$ ranging from 0 (straight edge) to 1 (semi-circular) in increment of 0.1 have been analyzed. For each crack shape, the crack depth ratio $\frac{a}{D}$ varied from 0.067 to 0.8 in eleven equal increments. The place ratio $\frac{x}{h}$ ranged from 0 (deepest point A in Figure 1) to 1 (surface intersection point $\mathrm{G}$ in Figure 1) in six equal increments. As a result, the stress intensities at 13 points on the crack front were evaluated for each crack front.

Detailed results are listed in Tables A1, A2 and A3 in the appendix for the end-free, endconstrained axial tension and the pure bending cases, respectively. Figure 3 compares some typical results for the end-free and end-constrained cases at the deepest point A (Figure 3a) 

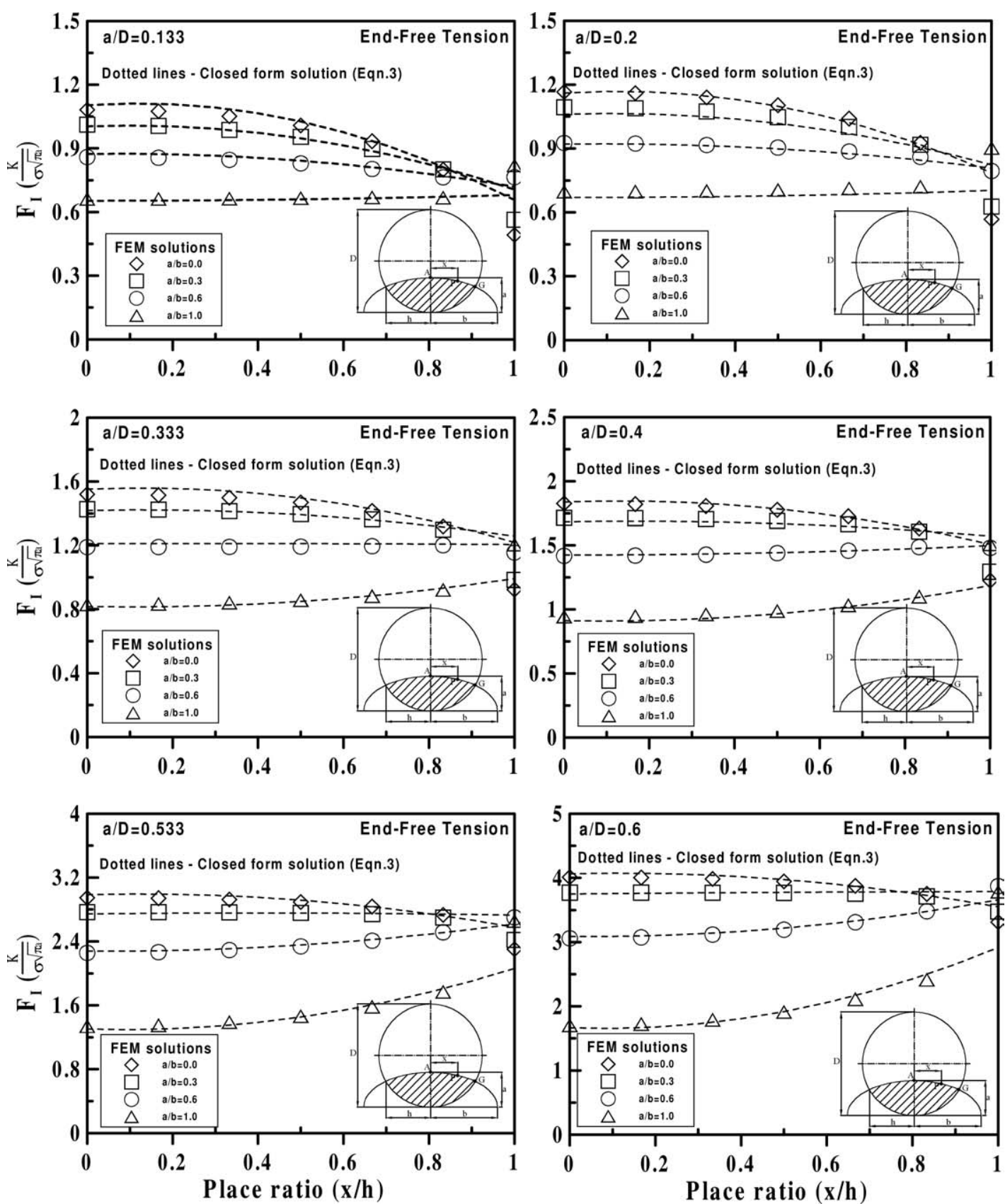

Figure 4. Geometry correction factor variation along the crack front. (a) under end-free axial tension; (b) under bending. 

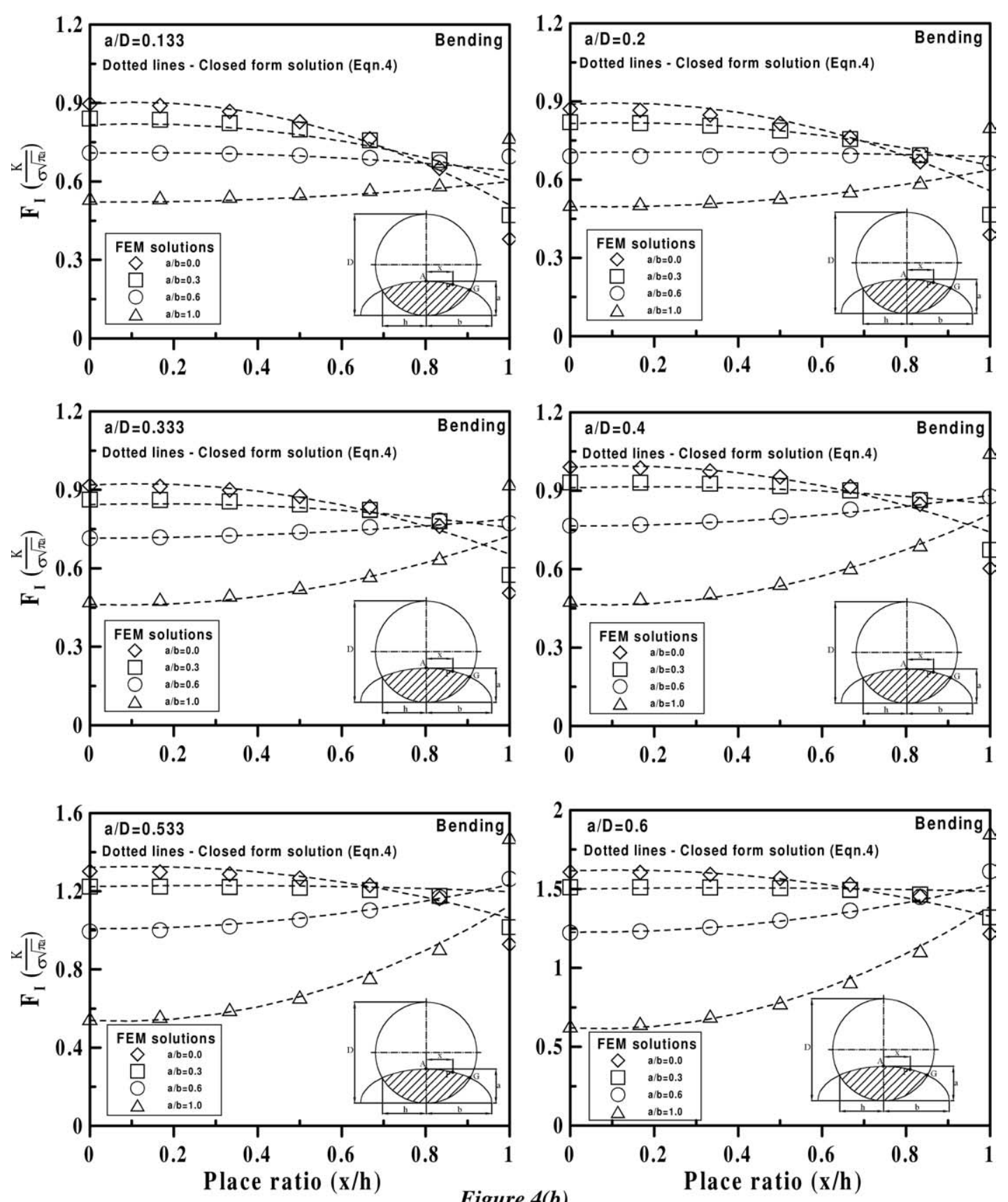

Figure 4. Continued.

and the surface point $\mathrm{G}$ (Figure $3 \mathrm{~b}$ ) for selected crack aspect ratios. It is clear that, for the same crack geometry, the stress intensity for the end-constrained case is always smaller than that for the end-free case. The discrepancy is within 5\% when the crack is small $\left(\frac{a}{D}<0.33\right)$. For deeper cracks, the discrepancy increases quickly to $25 \%$ at $\frac{a}{D}=0.6$ and $67 \%$ at $\frac{a}{D}=0.8$. 
This is reasonable as end constraint will impose a bending effect to check the crack opening and such an effect increases with the crack depth.

It has been pointed out that the square root singularity does not in general exist at the surface point G (Bazant and Estenssoro, 1979; Hayashi and Abe, 1980; Carpinteri and Brighenti, 1996). The usual meaning of stress intensity factor breaks down here. A plot of the geometry correction factor against the place ratio (see Figure 4) shows typically that the raw finite results at the surface point deviate markedly from the trends indicated by the interior points. Carpinteri and Brighenti (1996) pointed out that the region where the stress intensity breaks down is very small and proposed to take the result at a point next to the surface in the finite element mesh $\left(\frac{x}{h}=0.9\right)$. However, the present work showed that the stress intensities at $\frac{x}{h}=0.9$ may be up to $14 \%$ different from the stress intensities at the surface if the latter were deduced by extrapolating the trends exhibited by the interior points. In the current work, it is proposed to use a quadratic curve fitting to the interior points and obtain a pragmatic stress intensity value for the surface point by extrapolation. The raw finite element results deviate from these extrapolation results by $-27 \%$ to $43 \%$. The two results are within $6 \%$ of each other when $\frac{a}{b}=0.6$. Deviation increases as $\frac{a}{b}$ moves away from 0.6 in both directions. Incidentally, the square root singularity exists at the surface interception point of an elliptical crack with an aspect ratio of 0.6 when the Poisson ratio is around 0.3.

\section{Closed form stress intensity equations}

It has been pointed out before that a closed form equation that gives us the stress intensities at different points along the crack front for different crack geometries is very useful in evaluating practical fatigue life or stress corrosion life and assessing other structural integrity problems involving surface cracks in cylindrical rods.

By using a multi-parameter fitting technique, the geometry correction factor $F_{I}$ under tension at different points on the surface cracks with different aspect ratios and depth ratios may be expressed as:

$$
F_{I}=F_{I}\left(\frac{a}{b}, \frac{a}{D}, \frac{x}{h}\right)=\sum_{i=0}^{2} \sum_{j=0}^{7} \sum_{k=0}^{2} M_{i j k}\left(\frac{a}{b}\right)^{i}\left(\frac{a}{D}\right)^{j}\left(\frac{x}{h}\right)^{k}
$$

The coefficients $M_{i j k}$ for the end-free and end-constrained axial tension conditions are listed in Tables 1 and 2, respectively. For bending, the geometry correction factor $F_{I}$ may likewise be expressed as:

$$
F_{I}=F_{I}\left(\frac{a}{b}, \frac{a}{D}, \frac{x}{h}\right)=\sum_{i=0}^{2} \sum_{j=0}^{6} \sum_{k=0}^{2} N_{i j k}\left(\frac{a}{b}\right)^{i}\left(\frac{a}{D}\right)^{j}\left(\frac{x}{h}\right)^{k}
$$

The coefficients $N_{i j k}$ for bending are listed in Table 3. The $F_{I}$ values at the surface point G employed in the above fitting come from quadratic extrapolation as mentioned in the previous section. Figure 4 compares the closed form solution (dotted lines) with the raw finite element results (discrete symbols) for different values of place ratio, crack depth ratio and crack aspect ratio. The maximum deviation of the closed form values from the raw finite element data is within $3 \%$, whereas most of the deviations are within $1 \%$. 
Table 1. Coefficients $M_{i j k}$ in Equation 3 for end-free axial tension condition

\begin{tabular}{|c|c|c|c|c|c|c|c|c|c|}
\hline \multirow[b]{2}{*}{$j i$} & \multicolumn{3}{|c|}{$k=0$} & \multicolumn{3}{|c|}{$k=1$} & \multicolumn{3}{|c|}{$k=2$} \\
\hline & 0 & 1 & 2 & 0 & 1 & 2 & 0 & 1 & 2 \\
\hline 0 & 0.220 & -0.326 & 0.266 & 0.123 & 0.065 & 0.118 & -0.409 & 1.011 & -1.584 \\
\hline 1 & 28.513 & -3.780 & -9.118 & 0.511 & -6.878 & -3.515 & -9.764 & -3.946 & 45.562 \\
\hline 2 & -354.782 & 79.489 & 85.381 & -2.034 & 47.747 & 75.016 & 128.817 & 41.099 & -552.891 \\
\hline 3 & 2178.632 & -571.094 & -465.013 & -19.569 & -119.954 & -587.594 & -727.078 & -316.682 & 3322.477 \\
\hline 4 & -7140.202 & 1976.255 & 1475.911 & 144.435 & 14.769 & 2197.404 & 2201.067 & 1284.860 & -10812.317 \\
\hline 5 & 12957.447 & -3583.421 & -2794.532 & -359.284 & 423.169 & -4264.810 & -3732.813 & -2563.292 & 19328.127 \\
\hline 6 & -12227.977 & 3256.770 & 2878.868 & 393.518 & -661.610 & 4138.287 & 3343.521 & 2455.158 & -17829.715 \\
\hline 7 & 4721.868 & -1163.158 & -1261.348 & -159.206 & 306.176 & -1588.135 & -1240.214 & -880.302 & 6638.698 \\
\hline
\end{tabular}

Table 2. Coefficients $M_{i j k}$ in Equation 3 for end-constrained axial tension condition

\begin{tabular}{|c|c|c|c|c|c|c|c|c|c|}
\hline \multirow[b]{2}{*}{$j \quad i$} & \multicolumn{3}{|c|}{$k=0$} & \multicolumn{3}{|c|}{$k=1$} & \multicolumn{3}{|c|}{$k=2$} \\
\hline & 0 & 1 & 2 & 0 & 1 & 2 & 0 & 1 & 2 \\
\hline 0 & 1.095 & -1.177 & 0.725 & 0.113 & 0.271 & -0.388 & -0.896 & 0.904 & 0.008 \\
\hline 1 & -1.336 & 17.924 & -17.427 & 1.824 & -11.649 & 10.074 & 3.092 & 0.701 & -4.883 \\
\hline 2 & 13.108 & -137.252 & 134.652 & -21.709 & 98.358 & -80.088 & -4.197 & -32.641 & 55.092 \\
\hline 3 & -43.689 & 545.816 & -551.902 & 105.483 & -415.027 & 328.165 & -13.255 & 204.104 & -305.079 \\
\hline 4 & 134.868 & -1223.334 & 1239.493 & -271.225 & 982.713 & -772.921 & 51.548 & -568.407 & 916.962 \\
\hline 5 & -242.653 & 1541.587 & -1548.537 & 387.470 & -1329.634 & 1055.952 & -59.329 & 857.543 & -1545.428 \\
\hline 6 & 254.093 & -1006.656 & 969.388 & -290.024 & 961.893 & -784.581 & 13.481 & -657.659 & 1372.595 \\
\hline 7 & -108.196 & 264.206 & -227.132 & 88.387 & -288.565 & 245.798 & 10.854 & 191.570 & -485.556 \\
\hline
\end{tabular}

\section{Comparison with solutions from the literature}

A number of stress intensity factor solutions for surface crack in cylindrical rod under tension and bending exist in the literature. These solutions have been derived using finite element analyses (Raju and Newman, 1986; Astiz, 1986; Shiratori et al., 1986; Carpinteri, 1992b; Couroneau and Royer, 1998; Shih and Chen, 2002), boundary integral method (Athanassiadis et al., 1981), general weight function (Caspers et al., 1990) or approximate analyses (Douad et al., 1978; Douad and Cartwright, 1985; Bush, 1976, 1981; Murakami and Tsuru, 1987). Most of these solutions are only available for a limited range of crack shapes and all are for the end-free condition in case of axial tension. Moreover, only the stress intensities for the deepest point (A in Figure 1) and/or the surface point ( $G$ in Figure 1) are reported. Nevertheless, a number of solutions for certain common crack geometries are herein compared.

\subsection{END-FREE AXIAL TENSION RESULTS}

Figure 5 compares some published solutions with the current results for the end-free axial tension case. Regarding the stress intensity at the deepest point A, for a straight crack front $\left(\frac{a}{b}=0\right.$, see Figure 5a), all the solutions collected agree well with each other with two exceptions: beyond a crack depth ratio of 0.4 , the result deduced from an experimental compliance measurement (Bush, 1981) starts to deviate from others, and gives from $3 \%$ to $20 \%$ under- 
Table 3. Coefficients $N_{i j k}$ in Equation 4 under bending

\begin{tabular}{|c|c|c|c|c|c|c|c|c|c|}
\hline \multirow[b]{2}{*}{$j \quad i$} & \multicolumn{3}{|c|}{$k=0$} & \multicolumn{3}{|c|}{$k=1$} & \multicolumn{3}{|c|}{$k=2$} \\
\hline & 0 & 1 & 2 & 0 & 1 & 2 & 0 & 1 & 2 \\
\hline 0 & 1.346 & -0.640 & -0.022 & 0.190 & -0.347 & 0.175 & -0.926 & 1.399 & -0.454 \\
\hline 1 & -9.627 & 6.435 & 0.207 & -1.323 & 2.839 & -1.635 & 6.767 & -10.348 & 2.400 \\
\hline 2 & 82.244 & -36.062 & -22.436 & 8.317 & -18.649 & 9.091 & -42.734 & 71.260 & -4.388 \\
\hline 3 & -360.650 & 102.765 & 148.962 & -31.454 & 70.186 & -32.253 & 162.595 & -263.786 & -18.246 \\
\hline 4 & 841.678 & -151.830 & -426.773 & 66.389 & -142.227 & 60.188 & -345.453 & 531.560 & 110.187 \\
\hline 5 & -973.482 & 107.831 & 554.803 & -71.557 & 144.956 & -55.293 & 375.935 & -544.306 & -186.619 \\
\hline 6 & 449.146 & -27.262 & -276.533 & 31.022 & -58.870 & 19.041 & -165.151 & 225.705 & 108.877 \\
\hline
\end{tabular}

estimation. Secondly, beyond a crack depth ratio of 0.5 , the solution derived from a general weight function method (Caspers et al., 1990) deviates abnormally from the group trend, and is probably not reliable there. The deviation of the compliance solution is understandable as the experimental approach measured an average bulk intensity for the whole crack instead of pinpointed the point of interest. Nevertheless, such an average value is a good approximation for stress intensity at the deepest point when the crack depth ratio is smaller than 0.4. For an aspect ratio $\frac{a}{b}=0.6$ (Figure 5b), the majority of the collected solutions are within $10 \%$ of the present data. The largest discrepancies are only about $13 \%$ and occur in the finite element (FE) solution by Carpinteri (1992b) (at $a / D=0.3$ ) and the weight function solution (Caspers et al., 1990) (at $a / D=0.533$ ). For $a$ circular-arc crack front $\left(\frac{a}{b}=1.0\right.$ Figure $\left.5 \mathrm{c}\right)$, the weight function solution (Caspers et al., 1990) is markedly above the other solutions. The FE solution by Carpinteri (1992b) is also higher, while that by Raju and Newman (1986) is lower than the current results. In all three crack shapes above, the FE results by Couroneau and Royer (1998) and by Shiratori et al. (1986) nearly coincide with the present results. It should be pointed out that in the above comparison, due to the difference in the idealized crack front geometry and the difference in mesh refinement, the same crack aspect ratio will not guarantee exactly the same crack shape. Nevertheless, in the face of these differences, the worst-case discrepancies among these three stress intensity factor solutions for the deepest point is about $13 \%$.

Regarding the surface point $\mathrm{G}$, there is considerably more scatter among solutions for a straight-edge crack $\left(\frac{a}{b}=0\right.$, Figure 5d). The compliance solutions (Daoud et al., 1978; Bush, 1981) are markedly higher than other solutions. It has been pointed out above that the compliance of a cracked member only reflects the overall effect of a surface crack and so only gives us an average stress intensity over the whole crack front. Its deviation from other solutions indicates that more detailed analysis is needed to account for stress intensity variation along the surface crack front. Carpinteri (1992b) and Astiz's (1986) FE solutions indicated higher stress intensities at small crack depths $\left(\frac{a}{D}<0.2\right)$. At larger crack depths, discrepancies reduced and these two solutions merge with the current results. These two FE solutions are within $8 \%$ of the current results. Solution from the weight function method (Caspers et al., 1990) crossed the present results at $\frac{a}{D}=0.5$. It is up to $39 \%$ higher than the present results for $\frac{a}{D}<0.5$, and is up to $15 \%$ lower for $\frac{a}{D}>0.5$. Shih and Chen's data (2002) 

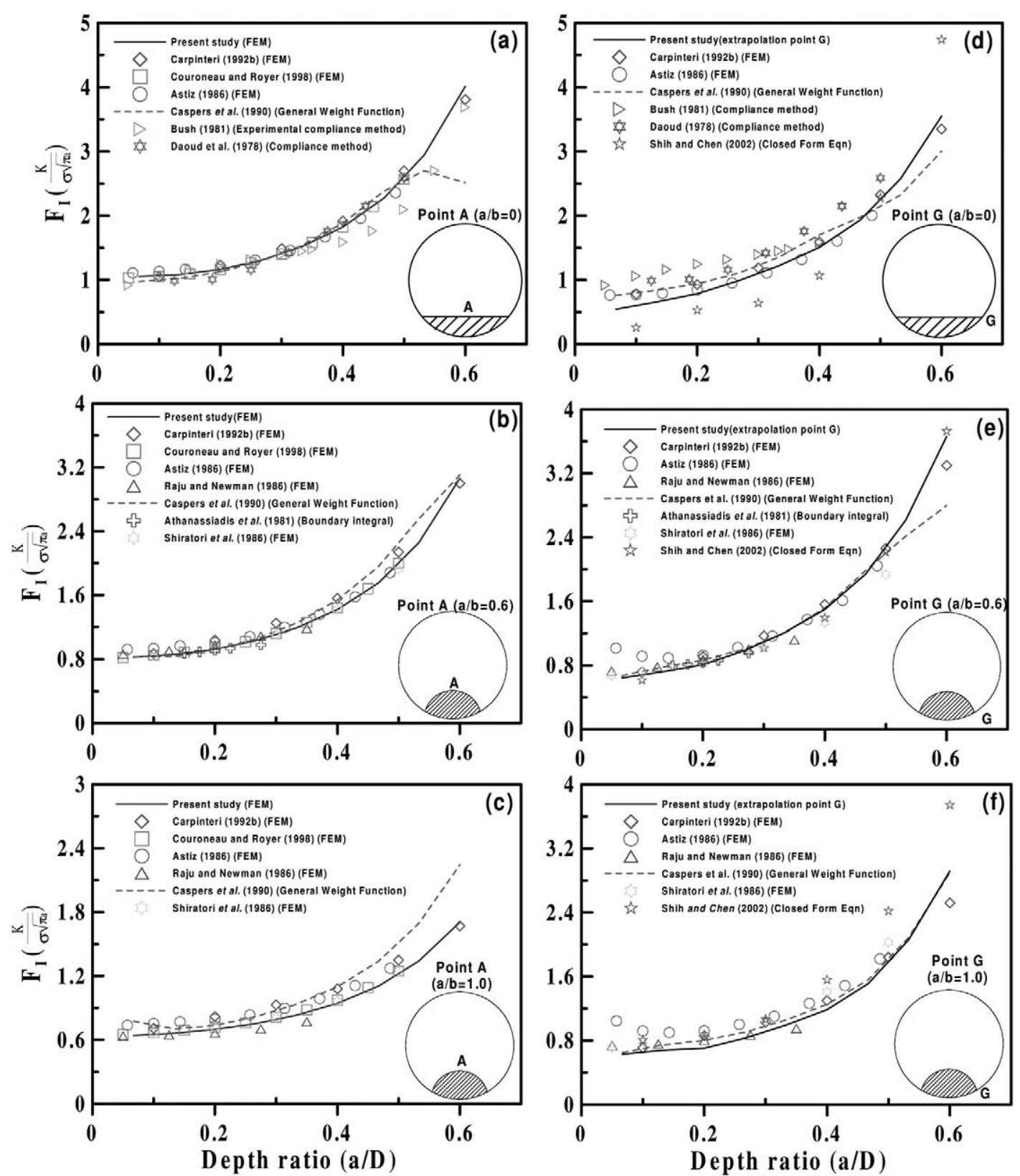

Figure 5. Comparison of different stress intensity solutions for an elliptical surface crack in a shaft under tension.

are lower than the current results for $\frac{a}{D}<0.5$ and higher than all other results for $\frac{a}{D}=0.6$. At aspect ratios of 0.6 and 1 (Figures 5e and 5f, respectively), most of the collected solutions are very close to the current solution. Astiz's (1986) FE results are prominently higher than other solutions at small crack depths $\left(\frac{a}{D}<0.2\right)$. At $\frac{a}{D}>0.5$, the weight function solution is lower than the FE results in the present study and by Carpinteri (1992b). On the other hand, 
Carpinteri's solution at $\frac{a}{D}=0.6$ is lower than the current solution. For $\frac{a}{b}=0.6$ and 1 , this difference amounts to $14 \%$.

In conclusion, for the deepest interior point $\mathrm{A}$, all the collected FE solutions (Raju and Newman, 1986; Astiz, 1986; Shiratori et al., 1986; Carpinteri, 1992b; Couroneau and Royer, 1998 ) agree well with the current results and the maximum discrepancy is $16 \%$. It should be pointed out that, although Shih and Chen (2002) has provided a rather complete closed form solution for this problem, their results for interior points are low and may even go negative, and so have not been included for comparison (Cai and Shin, 2004a). For the surface point G, agreement among different results are in general very good at $\frac{a}{b}=0.6$ where the square root singularity exists. For other aspect ratios, the overall agreement between several FE solutions (Raju and Newman, 1986; Astiz, 1986; Shiratori et al., 1986; Carpinteri, 1992b) with the current results is less good, with the maximum discrepancy equal to $32 \%$, which comes from Astiz's (1986) FE solutions for $\frac{a}{D}=0.05$.

\subsection{BENDING RESULTS}

Figure 6 compares current stress intensity results on bending with solutions collected from the literature. Regarding the stress intensity at the deepest point A, for a straight fronted crack (Figure 6a), considerable disagreement occurs at crack depth ratio $\frac{a}{D}$ below 0.3 , while all the results are close together for $\frac{a}{D}$ above 0.4. If only the solutions obtained through FE analysis are considered, then good agreement occurs throughout, and the literature results (Carpinteri, 1992b; Couroneau and Royer, 1998) are within $7.5 \%$ of the current results. For an aspect ratio $\frac{a}{b}$ of 0.6 (Figure 6b), all the results agree well, with the maximum deviation from the current results equal to $7.2 \%$. For circular-arc crack front $\left(\frac{a}{b}=1\right.$, Figure $\left.6 \mathrm{c}\right)$, most of the results are within $8 \%$ of the current solution except some of Carpinteri's (1992b) and Raju and Newman's (1986), which can be differ by up to $13 \%$ with the current results. The deviation of Raju and Newman's solution may be due to the fact that they used a circular instead of an elliptical crack. Shih and Chen (2002) 's closed form solution has not been included for comparison for the same reason as pointed out above in the tension case.

Regarding the surface point $\mathrm{G}$, for a straight fronted crack (Figure 6d), the compliance solutions are markedly above the current results. Carpinteri's FE results are 26\% higher at $\frac{a}{D}<0.1$ and $9.5 \%$ lower at $\frac{a}{D}=0.6$ but otherwise they agree well with the current work. The weight function results are close to the current solution at $\frac{a}{D} \geqslant 0.5$ but are markedly higher for smaller depth ratios. Shih and Chen (2002) are markedly lower than for $\frac{a}{D}<0.5$ and prominently higher than all other results for $\frac{a}{D}=0.6$. For an aspect ratio $\frac{a}{b}=0.6$ (Figure 6e), Carpinteri's solution (1992b) is close to the current results. Shiratori et al.'s FE solution (1986) is close to the weight function solution (Caspers et al., 1990) and both are below the current results at $\frac{a}{D} \geqslant 0.5$. Raju and Newman's FE solution (1986) is available for crack depth ratio below 0.4. Although Raju and Newman's results do not differ too much from the current solution, they do not show much variation as crack depth increases and this is 

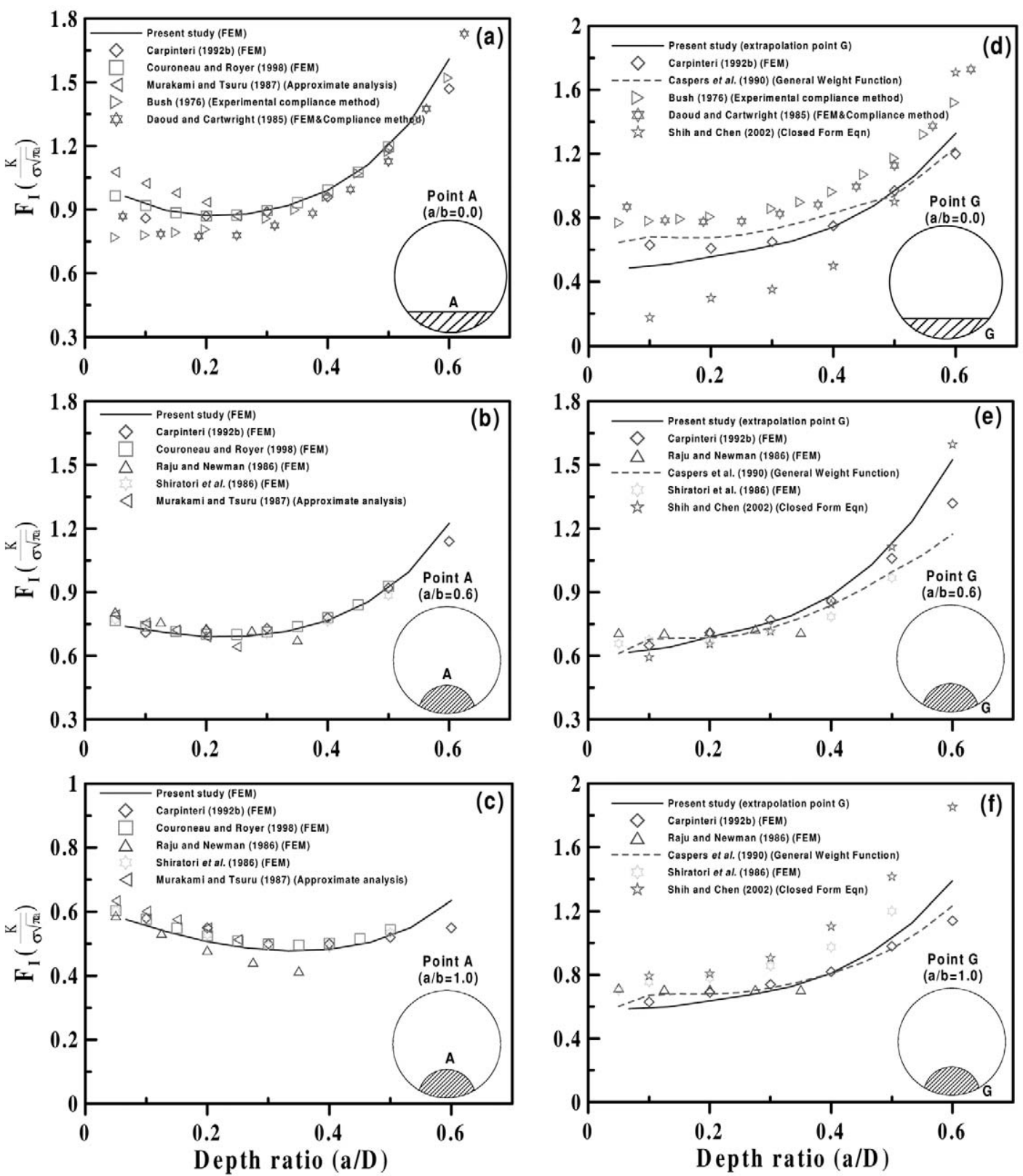

Figure 6. Comparison of different stress intensity solutions for an elliptical surface crack in a shaft under bending.

different from the trend shown by other solutions. Raju and Newman's solution has the same problem for $\frac{a}{b}=1$.

For circular-arc cracks $\frac{a}{b}=1$, Shih and Chen's results (2002) are higher than all other results. Shiratori et al.'s FE results (1986) are markedly above the current solutions. The weight function results (Caspers et al., 1990) and Carpinteri's results (1992b) are quite close to each other and are also close to the current results except at $\frac{a}{D}=0.6$. 
From the above comparisons, it can be seen that, except for some cases in Raju and Newman's (1986), Shiratori et al.'s (1986) and Shih and Chen's (2002), all stress intensity solutions derived directly from FE analysis have consistent trends and agree reasonably well with each other. The discrepancy exhibited by Raju and Newman's solution may be due to the circular crack shape they employed being different from the elliptical crack shapes used in other studies.

In conclusion, some remarks can be made. Firstly, different FE results for the interior points are in general in good mutual agreement. Secondly, the discrepancy among different solutions at the surface interception point is, in general, larger than that at the interior points. It has been pointed out that the square root singularity leading to the stress intensity concept does not in general exist at the surface interception point $\mathrm{G}$ of a surface crack. In the collected solutions, with the exception of Carpinteri (1992b), no special treatment to deal with this situation has been mentioned. Carpinteri computed the stress intensity for $0.0 \leqslant \frac{x}{h} \leqslant 0.9$ and ignored the results obtained for $0.9 \leqslant \frac{x}{h} \leqslant 1.0$. Experimental backtracking in the following section suggests that the stress intensity factor variation over the crack front follows a rather smooth curve in the near surface region. As a result, a quadratic fit to stress intensity values at interior positions is used in present study to obtain a pragmatic definition of surface stress intensity.

Lastly, Shih and Chen (2002) reported a closed form solution that is the most complete to date. It covers the whole crack front and for a wide range of crack aspect ratios and depth ratios. However, the present authors think that their solution is not completely valid (Cai and Shin, 2004a).

\section{Experimental verification of the stress intensity solutions}

In the above comparison with other numerical solutions, discrepancies to different degrees invariably exist. In order to assess more critically the current solution and, in particular, the validity of the present treatment at the surface point G, experimental analysis using the backtracking method (James et al., 1969; Schijve, 1985; Shin, 1990) has been attempted for the case of end-constrained axial tension. In this method, the crack growth rate $(\mathrm{da} / \mathrm{dN})$ versus the stress intensity range $(\Delta K)$ has first been obtained using standard specimens. Due to the variation in $\Delta K$, the amount of fatigue crack growth increment at different points on the crack front varies. By comparing successive crack profiles obtained after loading for a designated number of cycles, the crack growth rates at different points on the surface crack front can be evaluated. Knowing the crack growth rate at a particular point on the crack front, the associated stress intensity can then be deduced from the baseline da/dN versus $\Delta K$ data.

\subsection{EXPERIMENTAL PROCEDURES}

Standard compact tension (CT) specimens $(50 \mathrm{~mm}$ wide $\times 12 \mathrm{~mm}$ thick) conforming to ASTM E647 as well as rod specimens (15 $\mathrm{mm}$ in diameter and $200 \mathrm{~mm}$ long) were machined from an AISI 304 stainless steel plate stock. Semi-elliptical surface starter notches with different aspect ratios were created on the rod specimen using electric discharge machining with a thin circular copper foil electrode. Before testing, all specimens were stress relieved by a solution treated in vacuum at $1050{ }^{\circ} \mathrm{C}$ for one hour followed by quick cooling in a continuous supply of argon stream. 


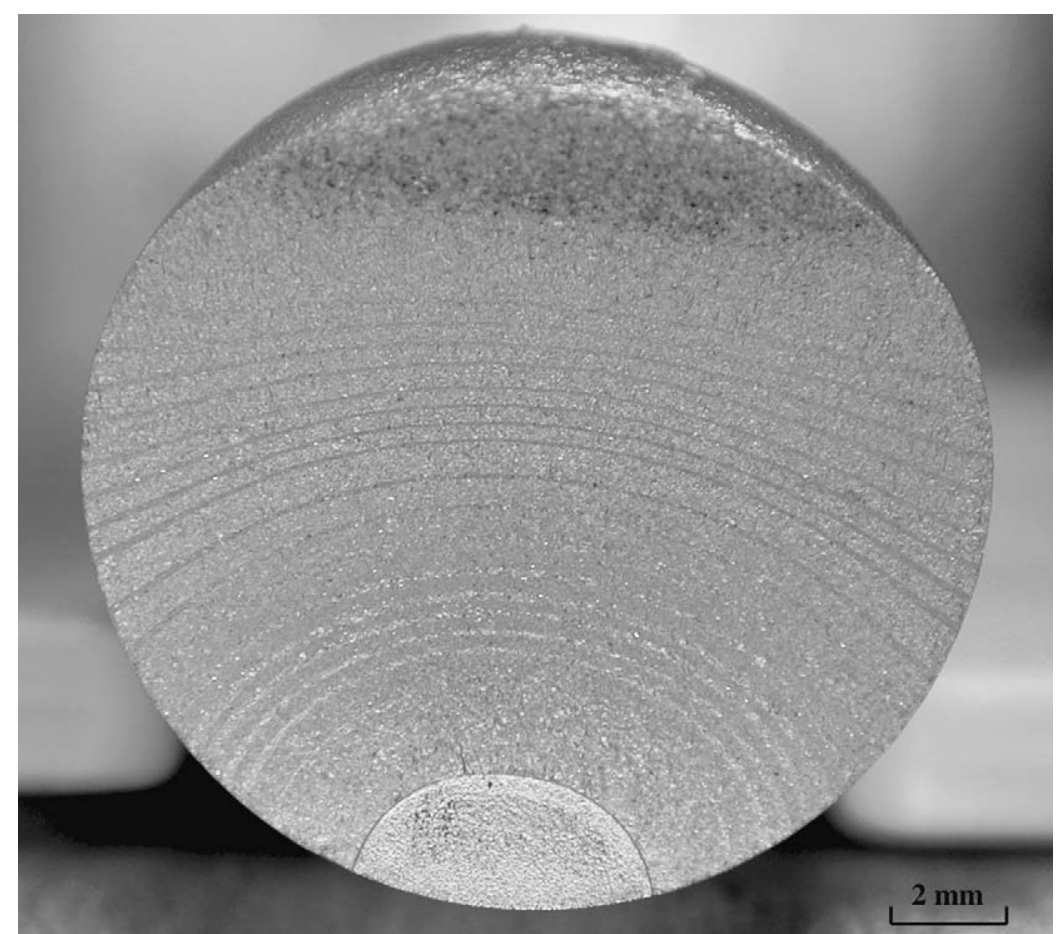

Figure 7. A typical rod fracture surface showing beach markings.

The CT specimens were tested under a loading with constant amplitude sinusoidal waveform at a frequency of $15 \mathrm{~Hz}$. Crack length was monitored with a traveling microscope to a resolution of $0.01 \mathrm{~mm}$.

The rod specimens were tested with a pair of rigid hydraulic grips to simulate the endconstrained condition. Surface crack length was monitored with a rotary traveling microscope to a resolution of $0.03 \mathrm{~mm}$. On a few specimens, instantaneous crack fronts were recorded periodically by creating beach marking using a waveform that has the same maximum but with the amplitude reduced to $30 \%$ of the instantaneous baseline loading. Constant amplitude as well as load shedding histories have been employed as the baseline loading. A typical beach marked fracture surface is shown in Figure 7. These instantaneous crack front records allow the crack growth rates and hence the stress intensities along the crack front to be deduced via the backtracking method. A normalized area-compliance method (Cai and Shin, 2004b) has also been employed on a number of rod specimens to monitor the surface crack growth to allow the crack size to be measured at much closer intervals and with much less effort than the beach marking technique. This compliance method provides an accurate definition of crack shape and depth (Cai and Shin, 2004b). Pre-matured crack closure invariably occurs during fatigue crack propagation. The closure behavior in a CT specimen is likely to be different from that in a rod specimen. Moreover, the phenomenon of crack closure is highly complex in a surface crack (Fleck et al., 1983; Fleck, 1988; Ray et al., 1987; Troha et al., 1988; Dawicke et al., 1990). Crack closure is heavier on the surface than in the interior. To ensure a valid backtracking stress intensity solution, the phenomenon of pre-mature crack closure has to be properly accounted for. In the current work, the compliance method aided with the offset procedure (Fleck, 1988) was employed to measure closure. A back face strain 


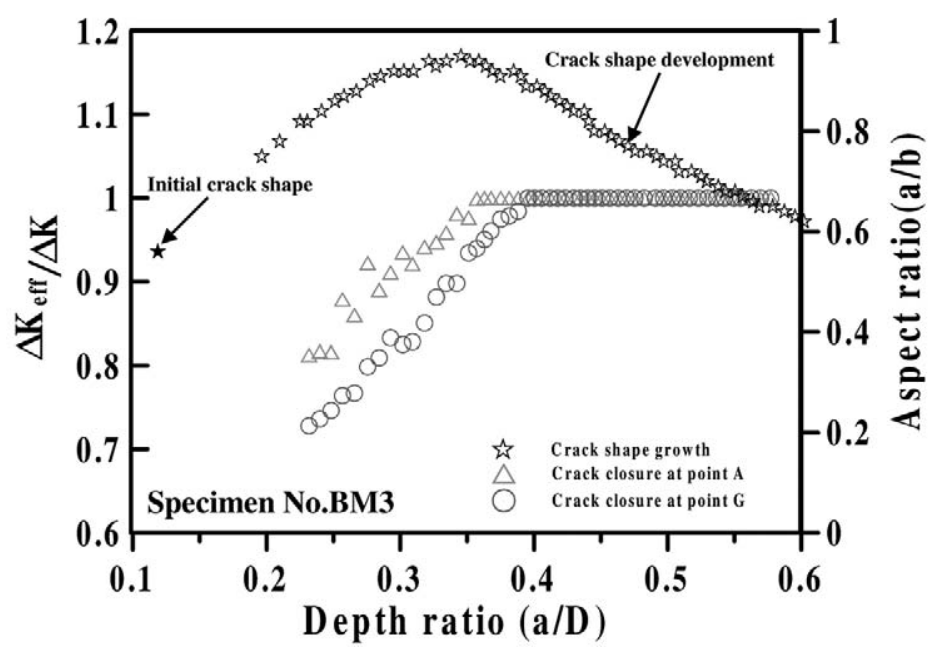

Figure 8. Typical development of the crack shape and the surface and interior closure responses in a surface cracked rod specimen.

gage was employed to monitor the closure response in a CT specimen. For the rod specimen, a strain gage straddling the crack at the tip region was used to reflect the crack closure on the surface. A back face gage, sticking in the middle of the uncracked surface, was used to reflect the bulk closure. This bulk closure is more representative of the closure in the interior (Fleck et al., 1983; Fleck 1988). On unloading, the slope of the back face compliance trace starts to change as closure at the surface begins to occur, and is continuously changing as closure spreads from the surface to the interior. The point on the compliance trace that corresponds to a fully closed crack is taken to determine the crack closure load for the deepest interior point (Dawicke et al., 1990). Crack closure at intermediate positions is approximated by a linear interpolation between the surface and the interior values.

\subsection{CRACK PROPAGATION RELATIONSHIP FROM STANDARD CT SPECIMENS}

The baseline crack growth data from standard fatigue crack propagation testing on CT specimens may be expressed as:

$$
\frac{d a}{d N}=9.947 \times 10^{-9}\left(\Delta K_{e f f}\right)^{2.973}
$$

where $\frac{d a}{d N}$ is the crack growth rate in $\mathrm{mm} /$ cycle and $\Delta K_{e f f}$ is the portion of applied stress intensity range during which the crack stays open. The unit of $\Delta K_{\text {eff }}$ is $\mathrm{MPa} \sqrt{ } \mathrm{m}$.

\subsection{EXPERIMENTAL RESULTS}

Figure 8 shows a typical crack shape development in one of the rod specimens under constant amplitude axial tension loading. The initial aspect ratio $\frac{a}{b}$ of the semi-elliptical starter notch was 0.6. As crack initiated and grew, the aspect ratio increased steadily to $\sim 0.95$ at a crack depth ratio $\frac{a}{D} 0.35$. Thereafter it fell steadily back to $\sim 0.6$ again. Figure 8 also shows the development of the closure responses in terms of $\Delta K_{\text {eff }} / \Delta K$. Closure is heavier on the 


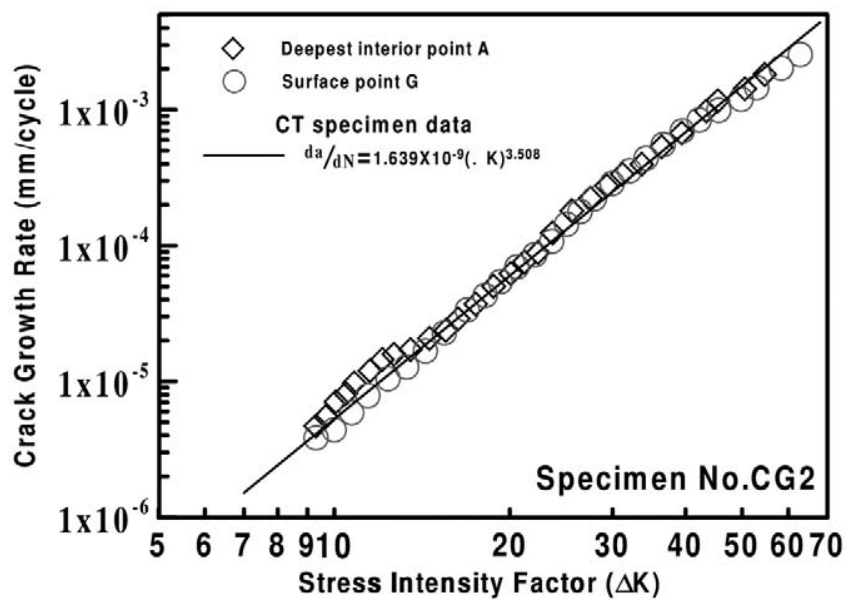

Figure 9. Comparison of typical crack growth responses at the deepest interior point A and the surface point G.

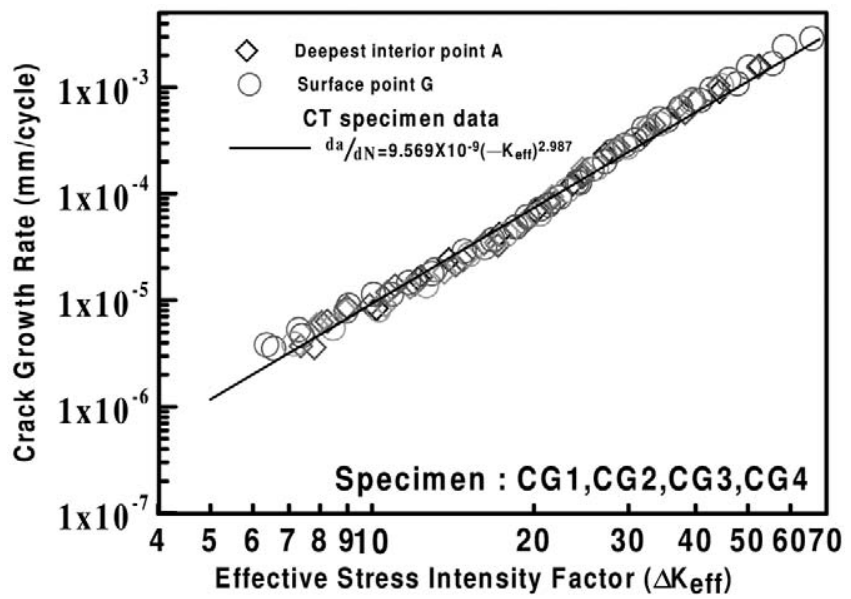

Figure 10. Comparison of the surface and interior growth in terms of the effective stress intensity range $\left(\Delta K_{e f f}\right)$.

surface than in the interior. As the crack grew, the degree of closure decreased. The surface crack became fully open everywhere during the whole loading cycle when the crack depth ratio reached 0.39 .

Figure 9 compares the typical crack growth responses at the deepest interior point $\mathrm{A}$ and the surface point $\mathrm{G}$ in terms of $\Delta K$ for one of the rod specimens. $\Delta K$ values are computed using Equation 3. Tests have been repeated on four rod specimens with different initial crack shapes, and reproducibility of the results is quite good. At lower $\Delta K$ values, crack growth in the interior is consistently faster than that at the surface. This may be attributed to the difference in crack closure responses as revealed in Figure 8. At higher $\Delta K s$ where the cracks were fully open everywhere during the whole loading cycle, crack growth responses at the two locations merge together. This consistency suggests the current interpolation treatment to obtain pragmatic stress intensity solutions at the surface point $G$ is acceptable.

Figure 10 compares the typical surface growth responses with the interior growth responses correlated in terms of the effective stress intensity range $\left(\Delta K_{\text {eff }}\right)$ for the rod specimens. Both the surface growth and interior growth fall in line with each other and with the CT specimen 

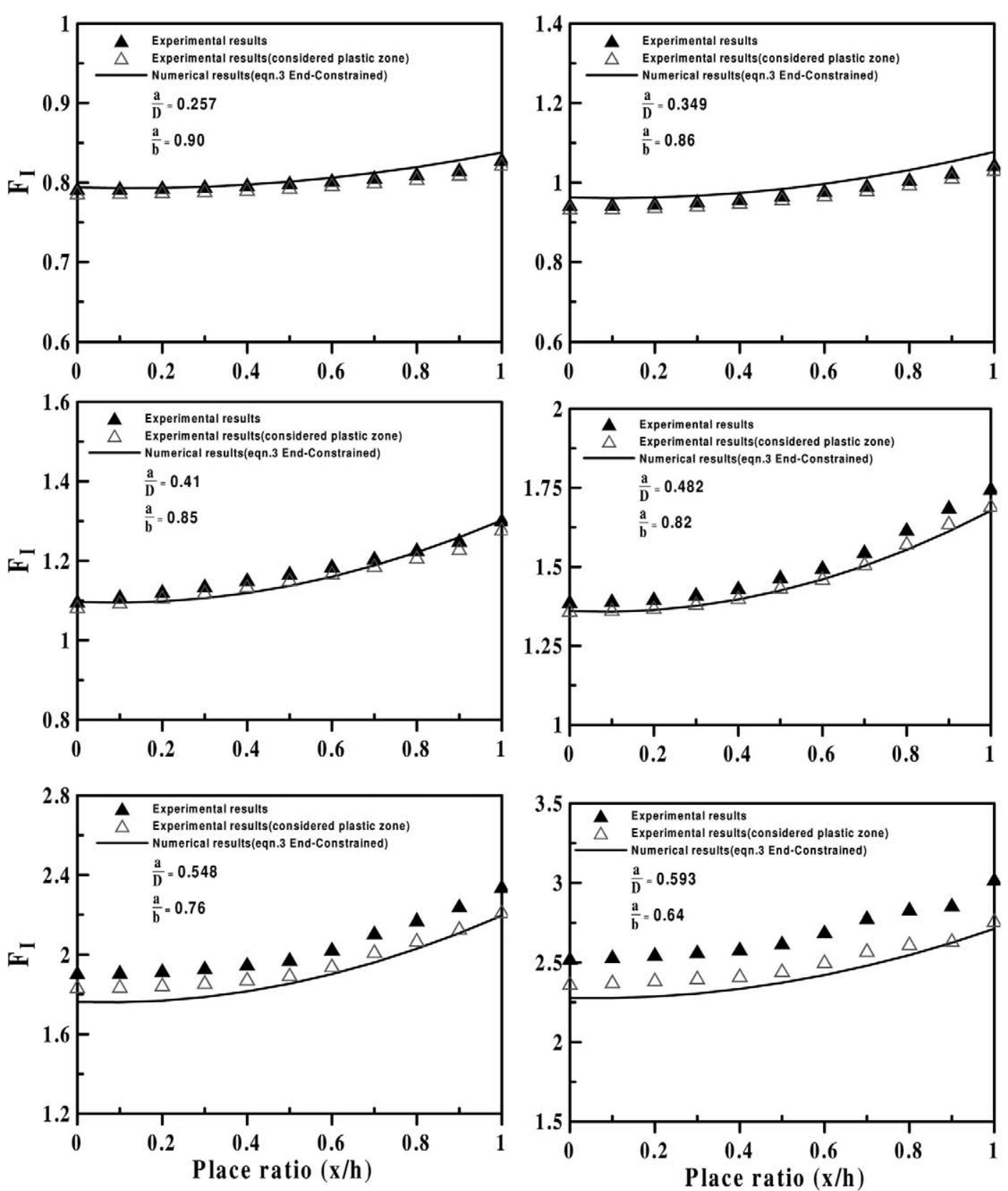

Figure 11. Comparison between the experimental backtracked and numerical stress intensity results along the surface crack front for a range of values of crack depth ratio and crack aspect ratio.

data throughout the whole range of $\Delta K_{\text {eff }}$ tested. Therefore, the current method to measure crack closure seems to be acceptable.

Figure 11 shows some typical comparison between the stress intensity values along the surface crack front obtained by experimental backtracking and the numerical results from Equation 3, for a range of crack depth and crack aspect ratios. The solid triangles represent direct backtracking deduction of stress intensity while the open triangles have accounted for 
the effect of plastic zone size correction on the stress intensity. For $\frac{a}{D}$ smaller than 0.482 , both the solid and dashed lines virtually overlap with each other. For $\frac{a}{D}$ between 0.257 and 0.41 , the numerical values are within $3 \%$ of the experimental values. For $\frac{a}{D}$ from 0.482 to 0.593 , the discrepancy between numerical values and the experimental values without accounting for plastic zone size effect steadily increases from $4.6 \%$ to $11 \%$, with experimental values consistently above numerical values. If a plastic zone size correction is applied during the backtracking calculation, the experimental results will move downward towards the numerical results (solid line), and the maximum error is only $8.5 \%$. It should be noted that the practical crack fronts are unlikely to be exactly the same as that modeled in the numerical analysis. Discrepancy in crack front shape increases as crack grows, leading to an increasing difference between numerical and experimental results. From the above comparison, it seems that the current closed form solution is adequate in describing the stress intensities along the whole crack front from surface to the interior of real surface cracks in cylindrical rods.

\section{Conclusion}

Experimental backtracking and finite element analysis have been performed to evaluate the stress intensities along the front of an elliptical surface crack in a cylindrical rod. Loading under end-free, end-constrained axial tension and pure bending have been analyzed using the finite element method. The solution covers crack aspect ratios from 0 to 1 and crack depth ratios from 0.067 to 0.8 . Convenient closed form stress intensity expressions for both loading cases have been given in terms of crack aspect ratio, crack depth ratio and place ratio. The maximum deviation of the closed form values from the raw finite element data is within 3\%, whereas most of the deviations are within $1 \%$.

The current closed form solution has been compared against a number of representative solutions collected from the literature. It has been found that, in general, different finite element results for the interior points are in good mutual agreement, while solutions derived from other methods may sometimes indicate different trends. Agreement is not so good at the surface interception point. This is believed to be caused by the different treatments or non-treatment to account for the absence of the square root singularity there. A quadratic extrapolation to the interior stress intensities to obtain a pragmatic stress intensity value for the surface point is adopted in the current work.

Experimental backtracking results corroborate well with the above closed form solution. It can be concluded that the present closed form stress intensity solution seems to be adequate in describing the stress intensities along the whole crack front from surface to the interior of real surface cracks in cylindrical rods.

\section{Acknowledgement}

The authors are grateful to the National Science Council, R.O.C. for financial support for this work through the projects NSC 91-2212-E-002-030 and NSC 92-2212-E-002-008. 


\section{Appendix. Numerical results for the geometry correction factor $F_{I}$ under axial tension and bending}

Table A1. Geometry correction factor $F_{I}$ for end-free axial tension condition

\begin{tabular}{|c|c|c|c|c|c|c|c|c|c|c|c|c|}
\hline \multirow[b]{2}{*}{$x / h$} & \multirow[b]{2}{*}{$a / D$} & \multicolumn{11}{|c|}{$a / b$} \\
\hline & & 0 & 0.1 & 0.2 & 0.3 & 0.4 & 0.5 & 0.6 & 0.7 & 0.8 & 0.9 & 1 \\
\hline \multirow[t]{12}{*}{ 0 } & 0.067 & 1.054 & 1.045 & 1.018 & 0.960 & 0.912 & 0.861 & 0.825 & 0.774 & 0.726 & 0.682 & 0.641 \\
\hline & 0.133 & 1.082 & 1.074 & 1.049 & 1.012 & 0.965 & 0.913 & 0.859 & 0.805 & 0.754 & 0.706 & 0.662 \\
\hline & 0.200 & 1.166 & 1.158 & 1.133 & 1.094 & 1.044 & 0.987 & 0.926 & 0.864 & 0.804 & 0.748 & 0.698 \\
\hline & 0.267 & 1.308 & 1.299 & 1.271 & 1.228 & 1.172 & 1.106 & 1.033 & 0.957 & 0.883 & 0.815 & 0.752 \\
\hline & 0.333 & 1.520 & 1.509 & 1.477 & 1.427 & 1.360 & 1.280 & 1.190 & 1.095 & 1.000 & 0.912 & 0.831 \\
\hline & 0.400 & 1.827 & 1.814 & 1.776 & 1.715 & 1.633 & 1.533 & 1.418 & 1.295 & 1.170 & 1.051 & 0.944 \\
\hline & 0.467 & 2.274 & 2.259 & 2.211 & 2.135 & 2.031 & 1.902 & 1.752 & 1.587 & 1.417 & 1.253 & 1.104 \\
\hline & 0.533 & 2.948 & 2.928 & 2.867 & 2.768 & 2.631 & 2.460 & 2.257 & 2.030 & 1.791 & 1.556 & 1.340 \\
\hline & 0.600 & 4.013 & 3.988 & 3.906 & 3.771 & 3.584 & 3.347 & 3.061 & 2.735 & 2.385 & 2.033 & 1.705 \\
\hline & 0.667 & 5.830 & 5.798 & 5.684 & 5.490 & 5.222 & 4.875 & 4.450 & 3.955 & 3.410 & 2.849 & 2.314 \\
\hline & 0.733 & 9.306 & 9.277 & 9.121 & 8.834 & 8.410 & 7.847 & 7.140 & 6.306 & 5.377 & 4.399 & 3.448 \\
\hline & 0.800 & 17.478 & 17.370 & 17.039 & 16.488 & 15.724 & 14.718 & 13.456 & 11.930 & 10.156 & 8.194 & 6.185 \\
\hline \multirow[t]{12}{*}{0.167} & 0.067 & 1.045 & 1.036 & 1.010 & 0.954 & 0.907 & 0.857 & 0.821 & 0.771 & 0.724 & 0.681 & 0.641 \\
\hline & 0.133 & 1.075 & 1.067 & 1.043 & 1.006 & 0.960 & 0.909 & 0.856 & 0.803 & 0.752 & 0.705 & 0.662 \\
\hline & 0.200 & 1.160 & 1.152 & 1.127 & 1.089 & 1.040 & 0.984 & 0.924 & 0.862 & 0.803 & 0.748 & 0.698 \\
\hline & 0.267 & 1.303 & 1.294 & 1.267 & 1.224 & 1.169 & 1.104 & 1.031 & 0.956 & 0.883 & 0.815 & 0.754 \\
\hline & $\mathbf{0 . 3 3 3}$ & 1.515 & 1.505 & 1.473 & 1.424 & 1.359 & 1.280 & 1.190 & 1.096 & 1.002 & 0.913 & 0.834 \\
\hline & 0.400 & 1.822 & 1.810 & 1.772 & 1.712 & 1.632 & 1.534 & 1.420 & 1.298 & 1.173 & 1.055 & 0.948 \\
\hline & 0.467 & 2.270 & 2.254 & 2.208 & 2.133 & 2.032 & 1.905 & 1.757 & 1.594 & 1.424 & 1.261 & 1.111 \\
\hline & $\mathbf{0 . 5 3 3}$ & 2.943 & 2.924 & 2.864 & 2.767 & 2.634 & 2.466 & 2.266 & 2.041 & 1.803 & 1.569 & 1.352 \\
\hline & 0.600 & 4.007 & 3.982 & 3.903 & 3.771 & 3.588 & 3.356 & 3.075 & 2.753 & 2.406 & 2.055 & 1.726 \\
\hline & 0.667 & 5.820 & 5.789 & 5.679 & 5.491 & 5.229 & 4.890 & 4.472 & 3.985 & 3.445 & 2.886 & 2.351 \\
\hline & 0.733 & 9.286 & 9.260 & 9.108 & 8.829 & 8.418 & 7.867 & 7.176 & 6.355 & 5.437 & 4.465 & 3.515 \\
\hline & 0.800 & 17.420 & 17.316 & 16.995 & 16.461 & 15.717 & 14.738 & 13.506 & 12.009 & 10.260 & 8.316 & 6.313 \\
\hline \multirow[t]{12}{*}{0.333} & 0.067 & 1.016 & 1.008 & 0.986 & 0.934 & 0.891 & 0.844 & 0.809 & 0.762 & 0.718 & 0.678 & 0.641 \\
\hline & 0.133 & 1.052 & 1.044 & 1.022 & 0.988 & 0.945 & 0.897 & 0.846 & 0.796 & 0.748 & 0.704 & 0.663 \\
\hline & 0.200 & 1.141 & 1.133 & 1.110 & 1.074 & 1.029 & 0.975 & 0.917 & 0.857 & 0.801 & 0.748 & 0.701 \\
\hline & 0.267 & 1.285 & 1.277 & 1.252 & 1.212 & 1.160 & 1.098 & 1.028 & 0.955 & 0.883 & 0.817 & 0.758 \\
\hline & 0.333 & 1.499 & 1.489 & 1.461 & 1.415 & 1.353 & 1.278 & 1.191 & 1.098 & 1.006 & 0.920 & 0.842 \\
\hline & 0.400 & 1.807 & 1.795 & 1.761 & 1.705 & 1.630 & 1.537 & 1.427 & 1.307 & 1.185 & 1.068 & 0.962 \\
\hline & 0.467 & 2.254 & 2.241 & 2.198 & 2.129 & 2.034 & 1.914 & 1.772 & 1.613 & 1.446 & 1.283 & 1.135 \\
\hline & 0.533 & 2.926 & 2.909 & 2.854 & 2.765 & 2.641 & 2.483 & 2.292 & 2.075 & 1.841 & 1.608 & 1.391 \\
\hline & 0.600 & 3.986 & 3.964 & 3.891 & 3.770 & 3.602 & 3.384 & 3.118 & 2.809 & 2.470 & 2.122 & 1.792 \\
\hline & 0.667 & 5.789 & 5.761 & 5.661 & 5.490 & 5.249 & 4.934 & 4.542 & 4.077 & 3.554 & 3.003 & 2.467 \\
\hline & 0.733 & 9.225 & 9.205 & 9.069 & 8.815 & 8.441 & 7.933 & 7.287 & 6.509 & 5.625 & 4.672 & 3.724 \\
\hline & 0.800 & 17.245 & 17.151 & 16.862 & 16.380 & 15.700 & 14.805 & 13.663 & 12.259 & 10.594 & 8.707 & 6.725 \\
\hline
\end{tabular}


Table A1. Continued.

\begin{tabular}{|c|c|c|c|c|c|c|c|c|c|c|c|c|}
\hline \multirow[b]{2}{*}{$x / h$} & \multirow[b]{2}{*}{$a / D$} & \multicolumn{11}{|c|}{$a / b$} \\
\hline & & 0 & 0.1 & 0.2 & 0.3 & 0.4 & 0.5 & 0.6 & 0.7 & 0.8 & 0.9 & 1 \\
\hline \multirow[t]{12}{*}{0.5} & 0.067 & 0.964 & 0.958 & 0.941 & 0.897 & 0.862 & 0.821 & 0.789 & 0.748 & 0.709 & 0.674 & 0.642 \\
\hline & 0.133 & 1.008 & 1.002 & 0.983 & 0.954 & 0.917 & 0.875 & 0.829 & 0.784 & 0.741 & 0.701 & 0.666 \\
\hline & 0.200 & 1.104 & 1.097 & 1.078 & 1.047 & 1.007 & 0.958 & 0.904 & 0.849 & 0.797 & 0.749 & 0.705 \\
\hline & 0.267 & 1.253 & 1.246 & 1.224 & 1.190 & 1.144 & 1.087 & 1.021 & 0.952 & 0.885 & 0.822 & 0.767 \\
\hline & 0.333 & 1.469 & 1.461 & 1.436 & 1.396 & 1.342 & 1.273 & 1.192 & 1.104 & 1.016 & 0.932 & 0.857 \\
\hline & 0.400 & 1.778 & 1.768 & 1.739 & 1.691 & 1.625 & 1.541 & 1.439 & 1.325 & 1.206 & 1.092 & 0.988 \\
\hline & 0.467 & 2.225 & 2.214 & 2.178 & 2.118 & 2.036 & 1.929 & 1.798 & 1.648 & 1.487 & 1.327 & 1.179 \\
\hline & 0.533 & 2.894 & 2.880 & 2.834 & 2.758 & 2.652 & 2.512 & 2.339 & 2.135 & 1.911 & 1.681 & 1.464 \\
\hline & 0.600 & 3.946 & 3.928 & 3.868 & 3.766 & 3.623 & 3.433 & 3.195 & 2.909 & 2.586 & 2.245 & 1.914 \\
\hline & 0.667 & 5.730 & 5.709 & 5.626 & 5.485 & 5.282 & 5.011 & 4.665 & 4.241 & 3.749 & 3.214 & 2.678 \\
\hline & 0.733 & 9.116 & 9.105 & 8.999 & 8.793 & 8.482 & 8.050 & 7.484 & 6.785 & 5.964 & 5.048 & 4.104 \\
\hline & 0.800 & 16.952 & 16.877 & 16.642 & 16.250 & 15.691 & 14.946 & 13.967 & 12.736 & 11.225 & 9.451 & 7.510 \\
\hline \multirow[t]{12}{*}{0.667} & 0.067 & 0.877 & 0.874 & 0.866 & 0.838 & 0.814 & 0.783 & 0.758 & 0.725 & 0.695 & 0.667 & 0.643 \\
\hline & 0.133 & 0.934 & 0.929 & 0.916 & 0.897 & 0.872 & 0.840 & 0.803 & 0.765 & 0.730 & 0.698 & 0.669 \\
\hline & 0.200 & 1.041 & 1.036 & 1.023 & 1.001 & 0.971 & 0.932 & 0.886 & 0.838 & 0.792 & 0.750 & 0.713 \\
\hline & 0.267 & 1.196 & 1.191 & 1.176 & 1.151 & 1.116 & 1.069 & 1.012 & 0.950 & 0.888 & 0.832 & 0.781 \\
\hline & 0.333 & 1.416 & 1.410 & 1.393 & 1.363 & 1.321 & 1.265 & 1.195 & 1.115 & 1.032 & 0.954 & 0.883 \\
\hline & 0.400 & 1.727 & 1.720 & 1.699 & 1.664 & 1.613 & 1.545 & 1.458 & 1.354 & 1.243 & 1.133 & 1.032 \\
\hline & 0.467 & 2.173 & 2.165 & 2.140 & 2.096 & 2.034 & 1.949 & 1.838 & 1.704 & 1.553 & 1.398 & 1.251 \\
\hline & 0.533 & 2.838 & 2.828 & 2.796 & 2.741 & 2.662 & 2.553 & 2.410 & 2.231 & 2.023 & 1.801 & 1.584 \\
\hline & 0.600 & 3.878 & 3.866 & 3.825 & 3.753 & 3.649 & 3.504 & 3.311 & 3.065 & 2.771 & 2.445 & 2.113 \\
\hline & 0.667 & 5.631 & 5.619 & 5.565 & 5.469 & 5.324 & 5.124 & 4.852 & 4.496 & 4.057 & 3.553 & 3.018 \\
\hline & 0.733 & 8.937 & 8.941 & 8.881 & 8.750 & 8.538 & 8.225 & 7.788 & 7.214 & 6.496 & 5.647 & 4.717 \\
\hline & 0.800 & 16.515 & 16.469 & 16.319 & 16.067 & 15.696 & 15.185 & 14.473 & 13.521 & 12.267 & 10.694 & 8.845 \\
\hline \multirow[t]{12}{*}{0.833} & 0.067 & 0.722 & 0.724 & 0.732 & 0.735 & 0.734 & 0.716 & 0.701 & 0.681 & 0.665 & 0.650 & 0.637 \\
\hline & 0.133 & 0.800 & 0.799 & 0.799 & 0.801 & 0.800 & 0.788 & 0.763 & 0.736 & 0.711 & 0.688 & 0.668 \\
\hline & 0.200 & 0.927 & 0.925 & 0.922 & 0.918 & 0.909 & 0.891 & 0.860 & 0.823 & 0.785 & 0.751 & 0.721 \\
\hline & 0.267 & 1.093 & 1.091 & 1.086 & 1.078 & 1.064 & 1.039 & 1.001 & 0.952 & 0.898 & 0.847 & 0.802 \\
\hline & 0.333 & 1.318 & 1.316 & 1.310 & 1.298 & 1.280 & 1.249 & 1.201 & 1.138 & 1.064 & 0.990 & 0.922 \\
\hline & 0.400 & 1.631 & 1.629 & 1.621 & 1.606 & 1.583 & 1.545 & 1.486 & 1.405 & 1.306 & 1.201 & 1.100 \\
\hline & 0.467 & 2.076 & 2.073 & 2.064 & 2.046 & 2.018 & 1.971 & 1.899 & 1.795 & 1.662 & 1.514 & 1.365 \\
\hline & 0.533 & 2.732 & 2.729 & 2.719 & 2.698 & 2.663 & 2.606 & 2.514 & 2.380 & 2.202 & 1.991 & 1.770 \\
\hline & 0.600 & 3.751 & 3.750 & 3.738 & 3.713 & 3.671 & 3.598 & 3.481 & 3.304 & 3.059 & 2.754 & 2.416 \\
\hline & 0.667 & 5.456 & 5.458 & 5.445 & 5.419 & 5.367 & 5.278 & 5.123 & 4.879 & 4.526 & 4.065 & 3.526 \\
\hline & 0.733 & 8.636 & 8.663 & 8.671 & 8.653 & 8.594 & 8.467 & 8.230 & 7.850 & 7.293 & 6.537 & 5.617 \\
\hline & 0.800 & 15.841 & 15.837 & 15.815 & 15.773 & 15.695 & 15.542 & 15.237 & 14.704 & 13.835 & 12.540 & 10.796 \\
\hline \multirow[t]{12}{*}{1} & 0.067 & 0.545 & 0.572 & 0.594 & 0.612 & 0.626 & 0.637 & 0.643 & 0.645 & 0.643 & 0.637 & 0.626 \\
\hline & 0.133 & 0.656 & 0.677 & 0.694 & 0.706 & 0.715 & 0.719 & 0.720 & 0.716 & 0.708 & 0.696 & 0.680 \\
\hline & 0.200 & 0.787 & 0.805 & 0.817 & 0.824 & 0.824 & 0.819 & 0.807 & 0.790 & 0.767 & 0.738 & 0.703 \\
\hline & 0.267 & 0.986 & 1.004 & 1.014 & 1.017 & 1.012 & 1.001 & 0.982 & 0.955 & 0.922 & 0.880 & 0.832 \\
\hline & 0.333 & 1.218 & 1.242 & 1.255 & 1.258 & 1.251 & 1.233 & 1.205 & 1.168 & 1.119 & 1.061 & 0.992 \\
\hline & 0.400 & 1.507 & 1.543 & 1.565 & 1.571 & 1.561 & 1.537 & 1.497 & 1.442 & 1.372 & 1.286 & 1.186 \\
\hline & 0.467 & 1.931 & 1.986 & 2.019 & 2.031 & 2.021 & 1.990 & 1.936 & 1.862 & 1.766 & 1.648 & 1.508 \\
\hline & 0.600 & 3.550 & 3.670 & 3.750 & 3.788 & 3.786 & 3.743 & 3.659 & 3.534 & 3.369 & 3.163 & 2.916 \\
\hline & 0.667 & 5.109 & 5.308 & 5.445 & 5.519 & 5.530 & 5.478 & 5.363 & 5.185 & 4.945 & 4.641 & 4.275 \\
\hline & 0.733 & 8.082 & 8.432 & 8.680 & 8.827 & 8.873 & 8.817 & 8.660 & 8.402 & 8.043 & 7.582 & 7.020 \\
\hline & 0.800 & 14.608 & 15.205 & 15.651 & 15.945 & 16.089 & 16.080 & 15.920 & 15.609 & 15.146 & 14.532 & 13.767 \\
\hline & 0.533 & 2.579 & 2.658 & 2.709 & 2.730 & 2.722 & 2.685 & 2.618 & 2.523 & 2.399 & 2.245 & 2.062 \\
\hline
\end{tabular}


Table A2. Geometry correction factor $F_{I}$ for end-constrained axial tension condition

\begin{tabular}{|c|c|c|c|c|c|c|c|c|c|c|c|c|}
\hline \multirow[b]{2}{*}{$x / h$} & \multirow[b]{2}{*}{$a / D$} & \multicolumn{11}{|c|}{$a / b$} \\
\hline & & 0 & 0.1 & 0.2 & 0.3 & 0.4 & 0.5 & 0.6 & 0.7 & 0.8 & 0.9 & 1 \\
\hline \multirow[t]{12}{*}{0} & 0.067 & 1.042 & 1.033 & 0.973 & 0.934 & 0.886 & 0.834 & 0.782 & 0.732 & 0.712 & 0.668 & 0.626 \\
\hline & 0.133 & 1.070 & 1.062 & 1.039 & 1.004 & 0.958 & 0.906 & 0.851 & 0.797 & 0.746 & 0.698 & 0.654 \\
\hline & 0.200 & 1.144 & 1.136 & 1.114 & 1.078 & 1.030 & 0.975 & 0.914 & 0.853 & 0.794 & 0.739 & 0.689 \\
\hline & 0.267 & 1.265 & 1.257 & 1.233 & 1.194 & 1.141 & 1.079 & 1.010 & 0.937 & 0.867 & 0.800 & 0.740 \\
\hline & 0.333 & 1.439 & 1.430 & 1.403 & 1.359 & 1.299 & 1.227 & 1.144 & 1.057 & 0.970 & 0.887 & 0.811 \\
\hline & 0.400 & 1.677 & 1.667 & 1.636 & 1.585 & 1.516 & 1.430 & 1.330 & 1.223 & 1.112 & 1.006 & 0.909 \\
\hline & 0.467 & 1.998 & 1.987 & 1.950 & 1.891 & 1.809 & 1.706 & 1.585 & 1.450 & 1.309 & 1.171 & 1.042 \\
\hline & 0.533 & 2.428 & 2.415 & 2.372 & 2.302 & 2.204 & 2.081 & 1.932 & 1.764 & 1.583 & 1.399 & 1.226 \\
\hline & 0.600 & 2.999 & 2.984 & 2.934 & 2.851 & 2.735 & 2.587 & 2.406 & 2.196 & 1.964 & 1.721 & 1.485 \\
\hline & 0.667 & 3.742 & 3.724 & 3.667 & 3.571 & 3.437 & 3.262 & 3.046 & 2.789 & 2.496 & 2.179 & 1.856 \\
\hline & 0.733 & 4.665 & 4.646 & 4.583 & 4.477 & 4.325 & 4.129 & 3.882 & 3.582 & 3.229 & 2.826 & 2.396 \\
\hline & 0.800 & 5.723 & 5.703 & 5.638 & 5.526 & 5.371 & 5.164 & 4.902 & 4.578 & 4.183 & 3.712 & 3.173 \\
\hline \multirow[t]{12}{*}{0.167} & 0.067 & 1.033 & 1.024 & 0.966 & 0.928 & 0.881 & 0.830 & 0.779 & 0.730 & 0.710 & 0.667 & 0.626 \\
\hline & 0.133 & 1.063 & 1.055 & 1.033 & 0.998 & 0.954 & 0.902 & 0.848 & 0.795 & 0.744 & 0.697 & 0.654 \\
\hline & 0.200 & 1.138 & 1.131 & 1.108 & 1.073 & 1.027 & 0.972 & 0.912 & 0.852 & 0.793 & 0.739 & 0.690 \\
\hline & 0.267 & 1.260 & 1.252 & 1.228 & 1.190 & 1.139 & 1.077 & 1.008 & 0.937 & 0.867 & 0.801 & 0.741 \\
\hline & 0.333 & 1.434 & 1.425 & 1.399 & 1.356 & 1.298 & 1.226 & 1.144 & 1.058 & 0.971 & 0.888 & 0.814 \\
\hline & 0.400 & 1.673 & 1.663 & 1.633 & 1.583 & 1.515 & 1.430 & 1.332 & 1.225 & 1.116 & 1.010 & 0.913 \\
\hline & 0.467 & 1.994 & 1.983 & 1.948 & 1.890 & 1.810 & 1.708 & 1.588 & 1.455 & 1.315 & 1.177 & 1.048 \\
\hline & 0.533 & 2.424 & 2.411 & 2.370 & 2.301 & 2.206 & 2.085 & 1.939 & 1.772 & 1.592 & 1.409 & 1.236 \\
\hline & 0.600 & 2.995 & 2.980 & 2.932 & 2.851 & 2.738 & 2.593 & 2.416 & 2.209 & 1.978 & 1.737 & 1.501 \\
\hline & 0.667 & 3.738 & 3.720 & 3.665 & 3.572 & 3.442 & 3.271 & 3.060 & 2.807 & 2.518 & 2.203 & 1.881 \\
\hline & 0.733 & 4.659 & 4.641 & 4.580 & 4.477 & 4.331 & 4.140 & 3.900 & 3.606 & 3.258 & 2.861 & 2.433 \\
\hline & 0.800 & 5.716 & 5.698 & 5.635 & 5.527 & 5.376 & 5.176 & 4.923 & 4.607 & 4.221 & 3.759 & 3.226 \\
\hline \multirow[t]{12}{*}{0.333} & 0.067 & 1.004 & 0.995 & 0.942 & 0.908 & 0.866 & 0.819 & 0.770 & 0.723 & 0.704 & 0.664 & 0.627 \\
\hline & 0.133 & 1.041 & 1.034 & 1.014 & 0.982 & 0.940 & 0.891 & 0.839 & 0.788 & 0.740 & 0.696 & 0.655 \\
\hline & 0.200 & 1.120 & 1.113 & 1.092 & 1.060 & 1.016 & 0.963 & 0.905 & 0.847 & 0.791 & 0.739 & 0.692 \\
\hline & 0.267 & 1.243 & 1.237 & 1.215 & 1.179 & 1.131 & 1.071 & 1.005 & 0.935 & 0.867 & 0.803 & 0.745 \\
\hline & 0.333 & 1.419 & 1.412 & 1.387 & 1.347 & 1.292 & 1.224 & 1.145 & 1.060 & 0.975 & 0.894 & 0.821 \\
\hline & 0.400 & 1.659 & 1.651 & 1.623 & 1.577 & 1.513 & 1.432 & 1.338 & 1.233 & 1.125 & 1.021 & 0.925 \\
\hline & 0.467 & 1.982 & 1.971 & 1.939 & 1.885 & 1.811 & 1.715 & 1.600 & 1.471 & 1.333 & 1.196 & 1.068 \\
\hline & 0.533 & 2.412 & 2.400 & 2.363 & 2.300 & 2.211 & 2.097 & 1.958 & 1.798 & 1.622 & 1.441 & 1.268 \\
\hline & 0.600 & 2.983 & 2.969 & 2.925 & 2.852 & 2.748 & 2.613 & 2.446 & 2.247 & 2.024 & 1.786 & 1.551 \\
\hline & 0.667 & 3.723 & 3.708 & 3.658 & 3.574 & 3.455 & 3.298 & 3.101 & 2.863 & 2.585 & 2.278 & 1.958 \\
\hline & 0.733 & 4.642 & 4.626 & 4.571 & 4.479 & 4.348 & 4.174 & 3.954 & 3.680 & 3.352 & 2.969 & 2.550 \\
\hline & 0.800 & 5.696 & 5.679 & 5.624 & 5.529 & 5.393 & 5.215 & 4.986 & 4.698 & 4.340 & 3.905 & 3.394 \\
\hline \multirow[t]{5}{*}{0.5} & 0.067 & 0.949 & 0.943 & 0.898 & 0.872 & 0.838 & 0.796 & 0.752 & 0.709 & 0.695 & 0.659 & 0.627 \\
\hline & 0.133 & 1.000 & 0.994 & 0.978 & 0.951 & 0.915 & 0.871 & 0.824 & 0.777 & 0.733 & 0.694 & 0.658 \\
\hline & 0.200 & 1.085 & 1.079 & 1.062 & 1.035 & 0.996 & 0.948 & 0.894 & 0.839 & 0.787 & 0.740 & 0.697 \\
\hline & 0.267 & 1.213 & 1.207 & 1.189 & 1.158 & 1.115 & 1.061 & 0.999 & 0.933 & 0.868 & 0.808 & 0.754 \\
\hline & 0.333 & 1.392 & 1.385 & 1.365 & 1.330 & 1.282 & 1.220 & 1.146 & 1.065 & 0.983 & 0.905 & 0.835 \\
\hline
\end{tabular}


Table A2. Continued.

\begin{tabular}{|c|c|c|c|c|c|c|c|c|c|c|c|c|}
\hline \multirow[b]{2}{*}{$x / h$} & \multirow[b]{2}{*}{$a / D$} & \multicolumn{11}{|c|}{$a / b$} \\
\hline & & 0 & 0.1 & 0.2 & 0.3 & 0.4 & 0.5 & 0.6 & 0.7 & 0.8 & 0.9 & 1 \\
\hline \multirow{19}{*}{0.667} & 0.400 & 1.634 & 1.627 & 1.603 & 1.563 & 1.508 & 1.435 & 1.348 & 1.248 & 1.144 & 1.042 & 0.949 \\
\hline & 0.467 & 1.958 & 1.949 & 1.922 & 1.876 & 1.812 & 1.727 & 1.621 & 1.499 & 1.366 & 1.232 & 1.106 \\
\hline & 0.533 & 2.389 & 2.379 & 2.348 & 2.295 & 2.219 & 2.119 & 1.994 & 1.844 & 1.676 & 1.499 & 1.328 \\
\hline & 0.600 & 2.958 & 2.948 & 2.912 & 2.851 & 2.764 & 2.647 & 2.499 & 2.318 & 2.107 & 1.877 & 1.643 \\
\hline & 0.667 & 3.696 & 3.684 & 3.644 & 3.576 & 3.477 & 3.345 & 3.175 & 2.962 & 2.706 & 2.413 & 2.099 \\
\hline & 0.733 & 4.609 & 4.597 & 4.554 & 4.481 & 4.375 & 4.233 & 4.048 & 3.812 & 3.519 & 3.164 & 2.761 \\
\hline & 0.800 & 5.659 & 5.646 & 5.604 & 5.530 & 5.423 & 5.283 & 5.097 & 4.859 & 4.554 & 4.167 & 3.694 \\
\hline & 0.067 & 0.856 & 0.853 & 0.818 & 0.807 & 0.786 & 0.755 & 0.720 & 0.686 & 0.680 & 0.653 & 0.628 \\
\hline & 0.133 & 0.930 & 0.927 & 0.917 & 0.900 & 0.873 & 0.838 & 0.798 & 0.759 & 0.722 & 0.689 & 0.660 \\
\hline & 0.200 & 1.025 & 1.022 & 1.010 & 0.991 & 0.962 & 0.923 & 0.877 & 0.828 & 0.783 & 0.741 & 0.704 \\
\hline & 0.267 & 1.159 & 1.156 & 1.143 & 1.121 & 1.088 & 1.044 & 0.990 & 0.931 & 0.872 & 0.817 & 0.769 \\
\hline & 0.333 & 1.343 & 1.338 & 1.324 & 1.299 & 1.262 & 1.212 & 1.148 & 1.075 & 0.999 & 0.926 & 0.860 \\
\hline & 0.400 & 1.588 & 1.583 & 1.567 & 1.538 & 1.496 & 1.438 & 1.364 & 1.274 & 1.177 & 1.079 & 0.989 \\
\hline & 0.467 & 1.914 & 1.909 & 1.890 & 1.858 & 1.809 & 1.743 & 1.655 & 1.547 & 1.424 & 1.295 & 1.171 \\
\hline & 0.533 & 2.347 & 2.341 & 2.319 & 2.282 & 2.228 & 2.151 & 2.050 & 1.920 & 1.767 & 1.599 & 1.428 \\
\hline & 0.600 & 2.915 & 2.909 & 2.885 & 2.844 & 2.784 & 2.698 & 2.582 & 2.431 & 2.244 & 2.029 & 1.798 \\
\hline & 0.667 & 3.650 & 3.643 & 3.618 & 3.575 & 3.508 & 3.417 & 3.291 & 3.122 & 2.904 & 2.639 & 2.336 \\
\hline & 0.733 & 4.557 & 4.551 & 4.526 & 4.482 & 4.417 & 4.325 & 4.198 & 4.025 & 3.792 & 3.488 & 3.116 \\
\hline & 0.800 & 5.607 & 5.601 & 5.578 & 5.538 & 5.477 & 5.396 & 5.281 & 5.125 & 4.906 & 4.606 & 4.203 \\
\hline \multirow[t]{12}{*}{0.833} & 0.067 & 0.694 & 0.696 & 0.665 & 0.685 & 0.692 & 0.682 & 0.665 & 0.646 & 0.648 & 0.634 & 0.620 \\
\hline & 0.133 & 0.796 & 0.796 & 0.799 & 0.801 & 0.799 & 0.785 & 0.759 & 0.731 & 0.705 & 0.682 & 0.662 \\
\hline & 0.200 & 0.914 & 0.914 & 0.913 & 0.910 & 0.902 & 0.884 & 0.853 & 0.816 & 0.778 & 0.743 & 0.713 \\
\hline & 0.267 & 1.062 & 1.062 & 1.059 & 1.053 & 1.041 & 1.018 & 0.981 & 0.933 & 0.881 & 0.831 & 0.787 \\
\hline & 0.333 & 1.254 & 1.253 & 1.249 & 1.241 & 1.225 & 1.198 & 1.154 & 1.096 & 1.027 & 0.958 & 0.895 \\
\hline & 0.400 & 1.506 & 1.504 & 1.499 & 1.489 & 1.471 & 1.439 & 1.389 & 1.318 & 1.231 & 1.138 & 1.047 \\
\hline & 0.467 & 1.836 & 1.834 & 1.828 & 1.817 & 1.797 & 1.762 & 1.705 & 1.622 & 1.514 & 1.390 & 1.264 \\
\hline & 0.533 & 2.270 & 2.269 & 2.263 & 2.251 & 2.230 & 2.192 & 2.130 & 2.036 & 1.905 & 1.746 & 1.573 \\
\hline & 0.600 & 2.838 & 2.838 & 2.833 & 2.823 & 2.803 & 2.766 & 2.701 & 2.598 & 2.447 & 2.249 & 2.018 \\
\hline & 0.667 & 3.570 & 3.571 & 3.568 & 3.562 & 3.545 & 3.515 & 3.455 & 3.352 & 3.190 & 2.959 & 2.664 \\
\hline & 0.733 & 4.475 & 4.477 & 4.478 & 4.477 & 4.472 & 4.454 & 4.411 & 4.326 & 4.175 & 3.932 & 3.588 \\
\hline & 0.800 & 5.526 & 5.530 & 5.535 & 5.541 & 5.546 & 5.549 & 5.533 & 5.486 & 5.378 & 5.175 & 4.837 \\
\hline \multirow[t]{12}{*}{1} & 0.067 & 0.506 & 0.528 & 0.548 & 0.565 & 0.580 & 0.593 & 0.603 & 0.611 & 0.617 & 0.620 & 0.621 \\
\hline & 0.133 & 0.653 & 0.675 & 0.691 & 0.703 & 0.711 & 0.714 & 0.712 & 0.705 & 0.694 & 0.679 & 0.659 \\
\hline & 0.200 & 0.794 & 0.813 & 0.826 & 0.833 & 0.834 & 0.829 & 0.819 & 0.803 & 0.781 & 0.753 & 0.719 \\
\hline & 0.267 & 0.954 & 0.974 & 0.986 & 0.991 & 0.987 & 0.976 & 0.958 & 0.931 & 0.897 & 0.855 & 0.806 \\
\hline & $\mathbf{0 . 3 3 3}$ & 1.151 & 1.176 & 1.191 & 1.195 & 1.190 & 1.173 & 1.147 & 1.110 & 1.062 & 1.005 & 0.936 \\
\hline & 0.400 & 1.400 & 1.435 & 1.455 & 1.462 & 1.456 & 1.435 & 1.401 & 1.354 & 1.292 & 1.217 & 1.128 \\
\hline & 0.467 & 1.723 & 1.770 & 1.800 & 1.812 & 1.806 & 1.783 & 1.742 & 1.684 & 1.608 & 1.514 & 1.402 \\
\hline & 0.533 & 2.145 & 2.209 & 2.251 & 2.272 & 2.270 & 2.246 & 2.200 & 2.132 & 2.041 & 1.929 & 1.795 \\
\hline & 0.600 & 2.695 & 2.781 & 2.840 & 2.873 & 2.879 & 2.859 & 2.813 & 2.740 & 2.641 & 2.515 & 2.363 \\
\hline & 0.667 & 3.402 & 3.511 & 3.591 & 3.642 & 3.663 & 3.656 & 3.620 & 3.554 & 3.460 & 3.337 & 3.184 \\
\hline & 0.733 & 4.287 & 4.412 & 4.511 & 4.583 & 4.627 & 4.645 & 4.636 & 4.601 & 4.538 & 4.449 & 4.333 \\
\hline & 0.800 & 5.348 & 5.467 & 5.570 & 5.657 & 5.729 & 5.784 & 5.824 & 5.849 & 5.857 & 5.849 & 5.826 \\
\hline
\end{tabular}


Table A3. Geometry correction factor $F_{I}$ for bending

\begin{tabular}{|c|c|c|c|c|c|c|c|c|c|c|c|c|}
\hline \multirow[b]{2}{*}{$x / h$} & \multirow[b]{2}{*}{$a / D$} & \multicolumn{11}{|c|}{$a / b$} \\
\hline & & 0 & 0.1 & 0.2 & 0.3 & 0.4 & 0.5 & 0.6 & 0.7 & 0.8 & 0.9 & 1 \\
\hline \multirow[t]{12}{*}{0} & 0.067 & 0.963 & 0.954 & 0.929 & 0.878 & 0.834 & 0.786 & 0.739 & 0.692 & 0.649 & 0.609 & 0.576 \\
\hline & 0.133 & 0.897 & 0.890 & 0.870 & 0.840 & 0.801 & 0.757 & 0.710 & 0.662 & 0.618 & 0.576 & 0.537 \\
\hline & 0.200 & 0.872 & 0.866 & 0.848 & 0.820 & 0.783 & 0.739 & 0.690 & 0.640 & 0.592 & 0.547 & 0.506 \\
\hline & 0.267 & 0.879 & 0.873 & 0.856 & 0.828 & 0.790 & 0.743 & 0.692 & 0.637 & 0.583 & 0.532 & 0.486 \\
\hline & 0.333 & 0.917 & 0.911 & 0.893 & 0.863 & 0.823 & 0.773 & 0.716 & 0.654 & 0.592 & 0.532 & 0.478 \\
\hline & 0.400 & 0.991 & 0.984 & 0.964 & 0.932 & 0.888 & 0.832 & 0.767 & 0.695 & 0.621 & 0.549 & 0.482 \\
\hline & 0.467 & 1.112 & 1.104 & 1.082 & 1.045 & 0.994 & 0.930 & 0.854 & 0.768 & 0.678 & 0.588 & 0.504 \\
\hline & 0.533 & 1.302 & 1.294 & 1.268 & 1.224 & 1.164 & 1.087 & 0.995 & 0.889 & 0.775 & 0.659 & 0.550 \\
\hline & 0.600 & 1.609 & 1.599 & 1.566 & 1.512 & 1.437 & 1.341 & 1.224 & 1.088 & 0.938 & 0.783 & 0.634 \\
\hline & 0.667 & 2.126 & 2.113 & 2.070 & 1.998 & 1.899 & 1.771 & 1.614 & 1.429 & 1.222 & 1.002 & 0.787 \\
\hline & 0.733 & 3.082 & 3.063 & 3.002 & 2.899 & 2.755 & 2.570 & 2.342 & 2.069 & 1.758 & 1.421 & 1.083 \\
\hline & 0.800 & 5.140 & 5.110 & 5.011 & 4.841 & 4.606 & 4.302 & 3.923 & 3.466 & 2.934 & 2.344 & 1.737 \\
\hline \multirow[t]{12}{*}{0.167} & 0.067 & 0.953 & 0.945 & 0.922 & 0.872 & 0.829 & 0.783 & 0.736 & 0.691 & 0.648 & 0.609 & 0.577 \\
\hline & 0.133 & 0.890 & 0.883 & 0.865 & 0.836 & 0.799 & 0.755 & 0.709 & 0.662 & 0.618 & 0.577 & 0.539 \\
\hline & 0.200 & 0.866 & 0.861 & 0.844 & 0.817 & 0.781 & 0.738 & 0.690 & 0.641 & 0.594 & 0.550 & 0.509 \\
\hline & 0.267 & 0.874 & 0.869 & 0.852 & 0.825 & 0.789 & 0.744 & 0.693 & 0.639 & 0.586 & 0.536 & 0.490 \\
\hline & 0.333 & 0.913 & 0.907 & 0.890 & 0.862 & 0.823 & 0.774 & 0.718 & 0.657 & 0.596 & 0.537 & 0.483 \\
\hline & 0.400 & 0.987 & 0.981 & 0.962 & 0.931 & 0.888 & 0.834 & 0.771 & 0.700 & 0.627 & 0.555 & 0.489 \\
\hline & 0.467 & 1.108 & 1.101 & 1.080 & 1.044 & 0.995 & 0.933 & 0.859 & 0.775 & 0.685 & 0.596 & 0.513 \\
\hline & 0.533 & 1.299 & 1.291 & 1.266 & 1.223 & 1.165 & 1.091 & 1.001 & 0.898 & 0.785 & 0.670 & 0.561 \\
\hline & 0.600 & 1.605 & 1.595 & 1.564 & 1.511 & 1.439 & 1.346 & 1.232 & 1.099 & 0.951 & 0.798 & 0.650 \\
\hline & 0.667 & 2.121 & 2.108 & 2.067 & 1.998 & 1.901 & 1.778 & 1.625 & 1.444 & 1.240 & 1.023 & 0.808 \\
\hline & 0.733 & 3.074 & 3.056 & 2.997 & 2.897 & 2.758 & 2.579 & 2.356 & 2.090 & 1.784 & 1.450 & 1.113 \\
\hline & 0.800 & 5.124 & 5.095 & 4.999 & 4.835 & 4.607 & 4.311 & 3.943 & 3.496 & 2.973 & 2.391 & 1.787 \\
\hline \multirow[t]{12}{*}{0.333} & 0.067 & 0.925 & 0.918 & 0.899 & 0.854 & 0.816 & 0.772 & 0.728 & 0.685 & 0.645 & 0.608 & 0.580 \\
\hline & 0.133 & 0.868 & 0.863 & 0.847 & 0.823 & 0.790 & 0.749 & 0.705 & 0.661 & 0.619 & 0.580 & 0.545 \\
\hline & 0.200 & 0.849 & 0.844 & 0.830 & 0.807 & 0.776 & 0.736 & 0.691 & 0.645 & 0.599 & 0.556 & 0.518 \\
\hline & 0.267 & 0.859 & 0.855 & 0.841 & 0.818 & 0.786 & 0.745 & 0.698 & 0.646 & 0.595 & 0.546 & 0.502 \\
\hline & 0.333 & 0.900 & 0.895 & 0.880 & 0.856 & 0.822 & 0.778 & 0.726 & 0.668 & 0.609 & 0.551 & 0.499 \\
\hline & 0.400 & 0.975 & 0.970 & 0.954 & 0.927 & 0.889 & 0.841 & 0.782 & 0.715 & 0.644 & 0.575 & 0.510 \\
\hline & 0.467 & 1.097 & 1.091 & 1.073 & 1.042 & 0.999 & 0.942 & 0.874 & 0.795 & 0.709 & 0.622 & 0.540 \\
\hline & 0.533 & 1.288 & 1.281 & 1.259 & 1.222 & 1.170 & 1.103 & 1.021 & 0.923 & 0.816 & 0.704 & 0.596 \\
\hline & 0.600 & 1.593 & 1.584 & 1.556 & 1.510 & 1.445 & 1.361 & 1.257 & 1.132 & 0.992 & 0.843 & 0.696 \\
\hline & 0.667 & 2.105 & 2.093 & 2.057 & 1.996 & 1.909 & 1.797 & 1.657 & 1.489 & 1.295 & 1.085 & 0.873 \\
\hline & 0.733 & 3.048 & 3.032 & 2.979 & 2.890 & 2.766 & 2.603 & 2.400 & 2.152 & 1.862 & 1.540 & 1.209 \\
\hline & 0.800 & 5.072 & 5.046 & 4.961 & 4.814 & 4.608 & 4.341 & 4.003 & 3.589 & 3.096 & 2.536 & 1.945 \\
\hline \multirow[t]{5}{*}{0.5} & 0.067 & 0.874 & 0.870 & 0.856 & 0.821 & 0.791 & 0.754 & 0.714 & 0.675 & 0.639 & 0.607 & 0.584 \\
\hline & 0.133 & 0.828 & 0.825 & 0.816 & 0.799 & 0.773 & 0.739 & 0.700 & 0.660 & 0.622 & 0.587 & 0.555 \\
\hline & 0.200 & 0.816 & 0.813 & 0.804 & 0.789 & 0.765 & 0.732 & 0.692 & 0.650 & 0.608 & 0.569 & 0.533 \\
\hline & 0.267 & 0.831 & 0.829 & 0.819 & 0.803 & 0.779 & 0.746 & 0.705 & 0.658 & 0.611 & 0.565 & 0.524 \\
\hline & 0.333 & 0.875 & 0.872 & 0.862 & 0.845 & 0.819 & 0.784 & 0.739 & 0.687 & 0.632 & 0.578 & 0.527 \\
\hline
\end{tabular}


Table A3. Continued.

\begin{tabular}{|c|c|c|c|c|c|c|c|c|c|c|c|c|}
\hline \multirow[b]{2}{*}{$x / h$} & \multirow[b]{2}{*}{$a / D$} & \multicolumn{11}{|c|}{$a / b$} \\
\hline & & 0 & 0.1 & 0.2 & 0.3 & 0.4 & 0.5 & 0.6 & 0.7 & 0.8 & 0.9 & 1 \\
\hline \multirow{19}{*}{0.667} & 0.400 & 0.953 & 0.950 & 0.938 & 0.918 & 0.890 & 0.851 & 0.801 & 0.742 & 0.676 & 0.610 & 0.547 \\
\hline & 0.467 & 1.076 & 1.072 & 1.058 & 1.035 & 1.002 & 0.957 & 0.900 & 0.830 & 0.751 & 0.668 & 0.588 \\
\hline & 0.533 & 1.267 & 1.262 & 1.246 & 1.217 & 1.177 & 1.123 & 1.054 & 0.969 & 0.870 & 0.765 & 0.660 \\
\hline & 0.600 & 1.569 & 1.563 & 1.542 & 1.506 & 1.456 & 1.388 & 1.300 & 1.192 & 1.064 & 0.923 & 0.780 \\
\hline & 0.667 & 2.074 & 2.066 & 2.037 & 1.990 & 1.921 & 1.830 & 1.713 & 1.567 & 1.392 & 1.195 & 0.990 \\
\hline & 0.733 & 3.000 & 2.987 & 2.947 & 2.876 & 2.776 & 2.644 & 2.474 & 2.260 & 2.001 & 1.701 & 1.380 \\
\hline & 0.800 & 4.978 & 4.958 & 4.890 & 4.774 & 4.607 & 4.389 & 4.107 & 3.751 & 3.313 & 2.796 & 2.226 \\
\hline & 0.067 & 0.790 & 0.790 & 0.786 & 0.770 & 0.753 & 0.725 & 0.693 & 0.661 & 0.632 & 0.606 & 0.591 \\
\hline & 0.133 & 0.763 & 0.763 & 0.762 & 0.757 & 0.744 & 0.721 & 0.690 & 0.657 & 0.625 & 0.596 & 0.570 \\
\hline & 0.200 & 0.762 & 0.762 & 0.760 & 0.756 & 0.744 & 0.723 & 0.693 & 0.658 & 0.622 & 0.588 & 0.558 \\
\hline & 0.267 & 0.785 & 0.785 & 0.782 & 0.777 & 0.765 & 0.745 & 0.715 & 0.677 & 0.635 & 0.595 & 0.558 \\
\hline & 0.333 & 0.835 & 0.834 & 0.830 & 0.823 & 0.811 & 0.790 & 0.758 & 0.716 & 0.668 & 0.619 & 0.573 \\
\hline & 0.400 & 0.916 & 0.915 & 0.910 & 0.901 & 0.887 & 0.863 & 0.829 & 0.782 & 0.725 & 0.665 & 0.607 \\
\hline & 0.467 & 1.041 & 1.039 & 1.033 & 1.022 & 1.004 & 0.977 & 0.937 & 0.883 & 0.815 & 0.741 & 0.665 \\
\hline & 0.533 & 1.231 & 1.229 & 1.221 & 1.206 & 1.184 & 1.151 & 1.103 & 1.037 & 0.955 & 0.860 & 0.760 \\
\hline & 0.600 & 1.529 & 1.526 & 1.515 & 1.495 & 1.466 & 1.424 & 1.363 & 1.281 & 1.175 & 1.050 & 0.914 \\
\hline & 0.667 & 2.022 & 2.017 & 2.002 & 1.975 & 1.934 & 1.877 & 1.796 & 1.686 & 1.543 & 1.369 & 1.174 \\
\hline & 0.733 & 2.919 & 2.913 & 2.889 & 2.847 & 2.786 & 2.701 & 2.584 & 2.425 & 2.215 & 1.954 & 1.652 \\
\hline & 0.800 & 4.822 & 4.812 & 4.772 & 4.701 & 4.596 & 4.454 & 4.259 & 3.995 & 3.644 & 3.199 & 2.671 \\
\hline \multirow[t]{12}{*}{0.833} & 0.067 & 0.643 & 0.649 & 0.663 & 0.680 & 0.686 & 0.671 & 0.651 & 0.631 & 0.613 & 0.598 & 0.594 \\
\hline & 0.133 & 0.650 & 0.654 & 0.666 & 0.682 & 0.694 & 0.690 & 0.672 & 0.650 & 0.627 & 0.607 & 0.588 \\
\hline & 0.200 & 0.669 & 0.672 & 0.681 & 0.694 & 0.706 & 0.707 & 0.694 & 0.670 & 0.642 & 0.615 & 0.590 \\
\hline & 0.267 & 0.704 & 0.707 & 0.714 & 0.725 & 0.736 & 0.738 & 0.728 & 0.705 & 0.673 & 0.639 & 0.606 \\
\hline & 0.333 & 0.762 & 0.764 & 0.771 & 0.780 & 0.789 & 0.792 & 0.783 & 0.760 & 0.724 & 0.682 & 0.640 \\
\hline & 0.400 & 0.849 & 0.851 & 0.856 & 0.864 & 0.872 & 0.874 & 0.866 & 0.841 & 0.800 & 0.749 & 0.694 \\
\hline & 0.467 & 0.976 & 0.978 & 0.983 & 0.990 & 0.996 & 0.998 & 0.988 & 0.960 & 0.913 & 0.850 & 0.779 \\
\hline & 0.533 & 1.164 & 1.166 & 1.171 & 1.177 & 1.182 & 1.182 & 1.169 & 1.138 & 1.081 & 1.003 & 0.909 \\
\hline & 0.600 & 1.454 & 1.456 & 1.460 & 1.465 & 1.469 & 1.467 & 1.450 & 1.410 & 1.340 & 1.238 & 1.111 \\
\hline & 0.667 & 1.928 & 1.930 & 1.933 & 1.937 & 1.938 & 1.933 & 1.910 & 1.857 & 1.764 & 1.625 & 1.444 \\
\hline & 0.733 & 2.779 & 2.782 & 2.784 & 2.786 & 2.783 & 2.771 & 2.735 & 2.659 & 2.525 & 2.319 & 2.043 \\
\hline & 0.800 & 4.564 & 4.567 & 4.569 & 4.565 & 4.554 & 4.528 & 4.464 & 4.338 & 4.116 & 3.769 & 3.288 \\
\hline \multirow[t]{12}{*}{1} & 0.067 & 0.486 & 0.523 & 0.553 & 0.578 & 0.596 & 0.609 & 0.616 & 0.618 & 0.613 & 0.603 & 0.587 \\
\hline & 0.133 & 0.510 & 0.548 & 0.579 & 0.604 & 0.623 & 0.635 & 0.641 & 0.640 & 0.633 & 0.619 & 0.599 \\
\hline & 0.200 & 0.557 & 0.596 & 0.629 & 0.654 & 0.673 & 0.684 & 0.689 & 0.686 & 0.677 & 0.660 & 0.637 \\
\hline & 0.267 & 0.600 & 0.640 & 0.673 & 0.699 & 0.717 & 0.728 & 0.732 & 0.728 & 0.717 & 0.699 & 0.674 \\
\hline & $\mathbf{0 . 3 3 3}$ & 0.654 & 0.695 & 0.729 & 0.755 & 0.774 & 0.784 & 0.788 & 0.783 & 0.771 & 0.751 & 0.724 \\
\hline & 0.400 & 0.742 & 0.786 & 0.822 & 0.850 & 0.869 & 0.880 & 0.882 & 0.877 & 0.862 & 0.840 & 0.809 \\
\hline & 0.467 & 0.877 & 0.926 & 0.966 & 0.996 & 1.017 & 1.028 & 1.029 & 1.022 & 1.004 & 0.978 & 0.941 \\
\hline & 0.533 & 1.062 & 1.118 & 1.163 & 1.197 & 1.220 & 1.231 & 1.232 & 1.222 & 1.200 & 1.168 & 1.124 \\
\hline & 0.600 & 1.326 & 1.393 & 1.446 & 1.485 & 1.511 & 1.524 & 1.524 & 1.510 & 1.483 & 1.443 & 1.389 \\
\hline & 0.667 & 1.755 & 1.838 & 1.904 & 1.953 & 1.985 & 2.000 & 1.998 & 1.979 & 1.943 & 1.890 & 1.820 \\
\hline & 0.733 & 2.544 & 2.659 & 2.749 & 2.816 & 2.859 & 2.878 & 2.873 & 2.844 & 2.791 & 2.714 & 2.614 \\
\hline & 0.800 & 4.138 & 4.317 & 4.458 & 4.560 & 4.625 & 4.651 & 4.639 & 4.589 & 4.500 & 4.374 & 4.209 \\
\hline
\end{tabular}




\section{References}

Astiz, M.A. (1986). An incompatible singular elastic element for two- and three-dimensional crack problems. International Journal of Fracture 31, 105-124.

Athanassiadis, Boissenot, J.M., Brevet, P., Francois, D. and Raharinaivo, A. (1981). Linear elastic fracture mechanics computations of cracked cylindrical tensioned bodies. International Journal of Fracture 17, 553-566.

Barsoum, R.S. (1976). On the use of isoparametric finite element $\mathrm{s}$ in linear fracture mechanics. International Journal for Numerical Methods in Engineering 10, 25-37.

Bazant, Z.P. and Estenssoro, L.F. (1979). Surface singularity and crack propagation. International Journal of Solids and Structures 15, 405-426.

Bush, J. (1976). Experimentally determined stress-intensity factors for single-edge-crack round bars loaded in bending. Experimental Mechanics 249-257.

Bush, J. (1981). Stress intensity factors for single-edge-crack solid and hollow round bars loaded in tension. Journal of Testing and Evaluation 9, 216-223.

Cai, C.Q. and Shin, C.S. (2004a). 'A discussion on "The stress intensity factor study of an elliptical cracked shaft' by Y.S. Shih and J.J. Chen, Nuclear Engineering and Design 227(3), 355-358.

Cai, C.Q. and Shin, C.S. (2004b). A normalized area-compliance method for monitoring the surface crack development in a cylindrical rod. unpublished results.

Carpinteri, A. (1992a). Stress intensity factors for straight-fronted edge cracks in round bars. Engineering Fracture Mechanics 42, 1035-1040.

Carpinteri, A. (1992b). Elliptical-arc surface cracks in round bars. Fatigue and Fracture of Engineering Materials and Structures 15, 1141-1153.

Carpinteri, A. and Brighenti, R. (1996). Part-through cracks in round bars under cyclic combined axial and bending loading. International Journal of Fatigue 18, 33-39.

Caspers, M., Mattheck, C. and Munz, D. (1990). Propagation of surface cracks in notched and unnotched rods. Surface-Crack Growth: Models, Experiments, and Structures ASTM STP 1060, 365-389.

Couroneau, N. and Royer, J. (1998). Simplified model for the fatigue growth analysis of surface cracks in round bars under mode I. International Journal of Fatigue 10, 711-718.

Daoud, O.E.K., Cartwright, D.J. and Carney, M. (1978). Strain-energy release rate for a single edge-cracked circular bar in tension. Journal of Strain Analysis 13, 83-89.

Daoud, O.E.K. and Cartwright, D.J. (1985). Strain energy release rate for a circular-arc edge crack in a bar under tension or bending. Journal of Strain Analysis 20, 53-58

Dawicke, D.S., Grandt, Jr., A.F. and Newman Jr, J.C. (1990). Three-dimensional crack closure behavior. Engineering Fracture Mechanics 36, 111-121.

Fleck, N.A., Smith, I.F.C. and Smith, R.A. (1983). Closure behaviour of surface cracks, Fatigue and Fracture of Engineering Materials and Structures 3, 225-239.

Fleck, N.A. (1988). Influence of stress state on crack growth retardation. Basic Questions in Fatigue ASTM STP 924, 157-183.

Forman, R.G. and Shivakumar, V. (1986). Growth behavior of surface cracks in the circumferential plane of solid and hollow cylinders. Fracture Mechanics: Seventeen volume. ASTM 905, 59-74.

Hayashi, K. and Abe, H. (1980). Stress intensity factors for a semi-elliptical crack in the surface of a semi-infinite solid. International Journal of Fracture 16, 275-285.

Hellen, T.K. (1975). On the method of virtual crack extension. International Journal for Numerical methods in Engineering 9, 187-207.

James, L.A. and Anderson, W.E. (1969). A Simple Experimental procedure for Stress Intensity Calibration. Engineering Fracture Mechanics 1, 565.

Lorentzen, T., Kjaer, N.E. and Henriksen, T.K. (1986). The application of fracture mechanics to surface cracks in shafts. Engineering Fracture Mechanics 23, 1005-1014.

Mackay, T.L. and Alperin, B.J. (1985). Stress intensity factors for fatigue cracking in high-strength bolts. Engineering Fracture Mechanics 21, 391-397.

Murakami, Y. and Tsuru, H. (1987). Stress-Intensity factor equations for semi-elliptical surface crack in a shaft under bending. Stress Intensity Factors Handbook, Vol II. Oxford: Pergamon Press 657-658.

Nord, K.J. and Chung, T.J. (1986). Fracture and surface flaws in smooth and threaded round bars. International Journal of Fracture 30, 47-55. 
Parks, D.M. (1974). A stiffness derivative finite element technique for determination of elastic crack tip stress intensity factor. International Journal of Fracture 10, 487-502.

Raju, I.S. and Newman, J.C. (1986). Stress- intensity Factors for circumferential surface cracks in pipes and rods under tension and bending loads. Fracture Mechanics: Seventeen volume. ASTM STP 905, 789-805.

Ray, S.K., Perez, R. and Grandt, Jr., A.F. (1987). Fatigue Crack Closure of Corner Cracks Located at Holes Loaded in Tension or Bending. Fatigue and Fracture of Engineering Materials and Structures 10, 239-250.

Schijve, J. (1985). Comparison Between Empirical and Calculated Stress Intensity Factors of Hole Edge cracks. Engineering Fracture Mechanics 22, 49-58.

Shih, Y.S. and Chen, J.J. (2002). The stress intensity factor study of an elliptical cracked shaft. Nuclear Engineering and Design 214, 137-145.

Shin, C.S. (1990). The stress intensity factors of corner cracks emanating from holes. Engineering Fracture Mechanics 37, 423-436.

Shin, C.S. and Chen, P.C. (2004). Fatigue crack propagation testing using subsized rotating bending specimens. In press in Nuclear Engineering and design.

Shiratori, M., Miyoshi, T., Sakai, Y. and Zhang, G.R. (1986). Analysis of stress intensity factors for surface cracks subjected to arbitrarily distributed surface stresses. Trans. Japan Soc. Mech. Engrs. 660-662.

Troha, W.A., Nicholas, T. and Grandt, Jr, A.F. (1988). Three dimensional aspects of fatigue crack closure in surface flaws in polymethylmethacrylate material. ASTM STP 982, 598-616.

Wilhem, D., FitzGerald, J., Carter, J. and Dittmer, D. (1982). An empirical approach to determining K for surface cracks. Proceedings of the $5^{\text {th }}$ international Conference on Fracture Research 11-21. 